\title{
Calabi energies of extremal toric surfaces
}

\author{
Claude LeBrun
}

\begin{abstract}
We derive a formula for the $L^{2}$ norm of the scalar curvature of any extremal Kähler metric on a compact toric manifold, stated purely in terms of the geometry of the corresponding moment polytope. The main interest of this formula pertains to the case of complex dimension 2 , where it plays a key role in construction of of Bach-flat metrics on appropriate 4-manifolds.
\end{abstract}

\section{Introduction}

In an audacious attempt to endow complex algebraic varieties with canonical Riemannian metrics, Eugenio Calabi [11] initiated a systematic study of the squared $L^{2}$-norm

$$
\mathcal{C}(g)=\int_{M} s^{2} d \mu
$$

of the scalar curvature, considered as a functional on the space of Kähler metrics $g$ on a given compact complex manifold $(M, J)$; here $s$ and $d \mu$ of course denote the scalar curvature and Riemannian volume form of the given metric $g$. Given a Kähler class $\Omega \in H^{1,1}(M, \mathbb{R}) \subset H^{2}(M, \mathbb{R})$, his aim was to minimize the functional $\mathcal{C}(g)$ among all Kähler metrics $g=\omega(\cdot, J \cdot)$ with Kähler class $[\omega]=\Omega$. Calabi showed that the Euler-Lagrange equation for this variational problem is equivalent to requiring that $\nabla^{1,0} s$ be a holomorphic vector field, and he introduced the terminology extremal Kähler metrics for the solutions of this equation. It was later shown [13] that any extremal Kähler metric on a compact complex manifold actually minimizes the Calabi energy (1.1) in its Kähler class. Moreover, when such a minimizer exists, it is actually unique in its Kähler class, modulo automorphisms of the complex manifold $[\mathbf{1 4}, \mathbf{2 0}, \mathbf{4 3}]$. Our knowledge of existence remains imperfect, but considerable progress $[\mathbf{2}, \mathbf{1 6}, \mathbf{2 1}]$ has recently been made in the toric case that is focus of the present paper. However, a relatively elementary argument $[\mathbf{3 9}]$ shows that the set of Kähler classes represented

Supported in part by NSF grants DMS-0905159 and DMS-1205953. 
by extremal Kähler metrics on a compact complex manifold $(M, J)$ is necessarily open in $H^{1,1}(M, \mathbb{R})$.

Rather than minimizing the squared $L^{2}$-norm of the scalar curvature, as in (1.1), one might be tempted to instead minimize the squared $L^{2}$-norm of, say, the Riemann curvature tensor or the Ricci tensor. However, Calabi also observed $[\mathbf{1 1}]$ that, after appropriate normalization, such functionals only differ from (1.1) by a constant depending on the Kähler class. In this respect, real dimension four occupies a privileged position; not only does (1.1) become scale invariant in this dimension, but the relevant constants only depend on the topology of $M^{4}$, and so are independent of the Kähler class in question. For example, the Riemann curvature $\mathcal{R}$ and the Ricci tensor $r$ satisfy

$$
\begin{aligned}
\int_{M}|\mathcal{R}|^{2} d \mu & =-8 \pi^{2}(\chi+3 \tau)(M)+\frac{1}{4} \mathcal{C}(g) \\
\int_{M}|r|^{2} d \mu & =-8 \pi^{2}(2 \chi+3 \tau)(M)+\frac{1}{2} \mathcal{C}(g)
\end{aligned}
$$

for any compact Kähler manifold $(M, g, J)$ of complex dimension 2, where $\chi(M)$ and $\tau(M)$ are respectively the Euler characteristic and signature of the compact oriented 4-manifold $M$. Similarly, the Weyl curvature $W$, which is the conformally invariant part of the Riemann tensor $\mathcal{R}$, satisfies

$$
\int_{M}|W|^{2} d \mu=-12 \pi^{2} \tau(M)+\frac{1}{12} \mathcal{C}(g)
$$

for any compact Kähler surface $(M, g, J)$. Thus, if a Kähler metric $g$ on $M^{4}$ is a critical point of any of these Riemannian functionals, considered as a function on the bigger space of all Riemannian metrics on $M$, it must, in particular, be an extremal Kähler metric. In connection with (1.2), this observation has interesting consequences, some of which will be touched on in this article.

The primary goal of this article is to calculate the Calabi energy of any extremal Kähler metric on any toric surface - that is, on any simply connected compact complex manifold of complex dimension two which carries a compatible effective action of the 2 -torus $T^{2}=S^{1} \times S^{1}$. Any Kähler class on a toric surface is represented by a $T^{2}$-invariant Kähler metric, and, relative to such a metric, the action is generated by two periodic Hamiltonian vector fields. This pair of Hamiltonians gives us an $\mathbb{R}^{2}$-valued moment map, under which the image of our complex surface is a convex polygon $P \subset \mathbb{R}^{2}$. Moreover, modulo translations and $S L(2, \mathbb{Z})$ transformations, the moment polygon $P$ only depends on the given the Kähler class. Euclidean area measure on the interior of $P$ then allows us to define a barycenter for $P$ and a moment-of-inertia matrix $\Pi$ of $P$ relative to this barycenter. The edges of $P$ have rational slope, and are therefore endowed with preferred rescalings $d \lambda$ of 1-dimensional Lebesgue measure, chosen so that intervals of unit length correspond to separation vectors which are indivisible elements 
of the integer lattice $\mathbb{Z}^{2}$. This allows us to also define a barycenter of the perimeter of $P$, and hence also a vector $\overrightarrow{\mathfrak{D}} \in \mathbb{R}^{2}$ connecting the barycenter of the interior to the barycenter of the perimeter. Combining these ingredients, we then obtain a convenient formula for the Calabi energy of any extremal toric surface:

TheOREm A. Let $(M, J, \Omega)$ be a toric surface with fixed Kähler class, and let $P$ be the associated moment polygon. Then any Kähler metric $g$ with Kähler form $\omega \in \Omega$ has scalar curvature $s$ satisfying

$$
\frac{1}{32 \pi^{2}} \int_{M} s^{2} d \mu_{g} \geq \frac{|\partial P|^{2}}{2}\left(\frac{1}{|P|}+\overrightarrow{\mathfrak{D}} \cdot \Pi^{-1} \overrightarrow{\mathfrak{D}}\right)
$$

with equality iff $g$ is an extremal Kähler metric. Here $|P|$ denotes the area of the interior of $P,|\partial P|$ is the $\lambda$-length of its boundary, $\Pi$ is the momentof-inertia matrix of $P$, and $\overrightarrow{\mathfrak{D}}$ is the vector joining the barycenter $P$ to the barycenter of $\partial P$.

We give two proofs of this result. Our first proof, which is specifically adapted to complex dimension 2 , can be found in $\S 5$ below. Then, in $\S 6$, we prove a generalization, Theorem B, which holds for toric manifolds of arbitrary complex dimension. However, both proofs crucially depend on a detailed understanding of both the Futaki invariant and toric manifolds. We have therefore found it useful to preface our main calculations with a careful exploration of the underpinnings of these ideas. The article then concludes with a discussion of examples that illustrate our current knowledge of Bachflat Kähler metrics.

\section{The Futaki Invariant}

If $\left(M^{2 m}, J\right)$ is a compact complex $m$-manifold of Kähler type, and if

$$
\mathfrak{h}=H^{0}\left(M, \mathcal{O}\left(T^{1,0} M\right)\right)
$$

is the associated Lie algebra of holomorphic fields on $M$, the Futaki invariant assigns an element $\mathfrak{F}(\Omega)$ of the Lie coalgebra $\mathfrak{h}^{*}$ to every Kähler class $\Omega$ on $(M, J)$. To construct this element, let $g$ be a Kähler metric, with Kähler class $[\omega]=\Omega$, scalar curvature $s$, Green's operator $\mathcal{G}$, and volume form $d \mu$. We then define the Futaki invariant

$$
\mathfrak{F}(\Omega): H^{0}\left(M, \mathcal{O}\left(T^{1,0} M\right)\right) \longrightarrow \mathbb{C}
$$

to be the linear functional

$$
\Xi \longmapsto-2 \int_{M} \Xi(\mathcal{G} s) d \mu
$$

It is a remarkable fact, due to Futaki $[\mathbf{2 4}]$, Bando [6], and Calabi [12], that $\mathfrak{F}(\Omega)$ only depends on the Kähler class $\Omega$, and not on the particular metric $g$ chosen to represent it.

We will now assume henceforth that $b_{1}(M)=0$. Since $(M, J)$ is of Kähler type, the Hodge decomposition then tells us that $H^{0,1}(M)=0$, and 
it therefore follows $[\mathbf{1 2}, \mathbf{4 0}]$ that every holomorphic vector field $\Xi$ on $M$ can be written as $\nabla^{1,0} f$ for some smooth function $f=f_{\Xi}$, called a holomorphy potential. This allows us to re-express the Futaki invariant as

$$
\mathfrak{F}(\Xi, \Omega):=[\mathfrak{F}(\Omega)](\Xi)=-\int_{M}(s-\bar{s}) f_{\Xi} d \mu
$$

where $\bar{s}$ denotes the average value of the scalar curvature, which can be computed by the topological formula

$$
\bar{s}=4 \pi m \frac{c_{1} \cdot \Omega^{m-1}}{\Omega^{m}} .
$$

Of course, the negative sign appearing in (2.1) is strictly a matter of convention, and is used here primarily to ensure consistency with [40]. Also note that the $\bar{s}$ term in (2.1) could be dropped if one required that the holonomy potential $f_{\Xi}$ be normalized to have integral zero; however, we will find it useful to avoid systematically imposing such a normalization.

Let $\mathbf{H}$ now denote the identity component of the automorphism group of $(M, J)$, so that $\mathfrak{h}$ is its Lie algebra. Because the assumption that $b_{1}(M)=0$ implies that $\mathbf{H}$ is a linear algebraic group $[\mathbf{2 2}]$, we can define its unipotent radical $\mathbf{R}_{\mathbf{u}}$ to consist of the unipotent elements of its maximal solvable normal subgroup. If $\mathbf{G} \subset \mathbf{H}$ is a maximal compact subgroup, and if $\mathbf{G}_{\mathbb{C}} \subset \mathbf{H}$ is its complexification, then $\mathbf{G}_{\mathbb{C}}$ projects isomorphically onto the quotient group $\mathbf{H} / \mathbf{R}_{\mathbf{u}}$. The Chevalley decomposition $[\mathbf{1 7}]$ moreover expresses $\mathbf{H}$ as a semi-direct product

$$
\mathbf{H}=\mathbf{G}_{\mathbb{C}} \ltimes \mathbf{R}_{\mathbf{u}}
$$

and we have a corresponding split short exact sequence

$$
0 \rightarrow \mathfrak{r}_{\mathfrak{u}} \rightarrow \mathfrak{h} \rightarrow \mathfrak{g}_{\mathbb{C}} \rightarrow 0
$$

of Lie algebras.

In their pioneering work on extremal Kähler vector fields [25], Futaki and Mabuchi next restricted the Futaki invariant $\mathfrak{F}$ to $\mathfrak{g}_{\mathbb{C}} \subset \mathfrak{h}$. However, under mild hypotheses, this is not actually necessary:

Proposition 2.1. Let $\left(M^{2 m}, J\right)$ be a compact complex m-manifold of Kähler type for which $h^{1,0}=h^{2,0}=0$. Then the Futaki invariant $\mathfrak{F}(\Omega) \in \mathfrak{h}^{*}$ automatically annihilates the Lie algebra $\mathfrak{r}_{\mathfrak{u}}$ of the unipotent radical, and so belongs to $\mathfrak{g}_{\mathbb{C}}^{*}$. Moreover, this element is automatically real, and so belongs to $\mathfrak{g}^{*}$.

As we show in Appendix A, this is actually a straightforward consequence of a theorem of Nakagawa [45].

Because the Futaki invariant is invariant under biholomorphisms, it is unchanged by the action of $\mathbf{H}$ on $\mathfrak{h}$. It follows that $\mathfrak{F}(\Omega)$ must vanish when restricted to the derived subalgebra $[\mathfrak{h}, \mathfrak{h}]$. Thus, $\mathfrak{F}(\Omega): \mathfrak{h} \rightarrow \mathbb{C}$ is actually a Lie-algebra character. In particular, $\mathfrak{F}(\Omega)$ annihilates the derived subalgebra $[\mathfrak{g}, \mathfrak{g}]$ of the maximal compact. Since the compactness of $\mathbf{G}$ implies that it is a reductive Lie group, $\mathfrak{g}=[\mathfrak{g}, \mathfrak{g}] \oplus \mathfrak{z}$, where $\mathfrak{z}$ is the center of $\mathfrak{g}$. We thus conclude 
that $\mathfrak{F}(\Omega) \in \mathfrak{z}^{*}$ for any Kähler class $\Omega$ whenever $M$ is as in Proposition A.3. Since $\mathfrak{z}$ is contained in the Lie algebra of any maximal torus $\mathbf{T} \subset \mathbf{G}$, we thus deduce the following important fact:

Proposition 2.2. Let $\left(M^{2 m}, J\right)$ be a compact complex m-manifold of Kähler type for which $h^{1,0}=h^{2,0}=0$. Let $\mathbf{T}$ be a maximal torus in $\operatorname{Aut}(M, J)$, and let $\mathfrak{t}$ be the Lie algebra of $\mathbf{T}$. Then, for any Kähler class $\Omega$ on $M$, the Futaki invariant $\mathfrak{F}(\Omega)$ naturally belongs to $\mathfrak{t}^{*}$. In particular, $\mathfrak{F}(\Omega)$ is completely determined by its restriction to $\mathfrak{t}$.

Now, for a fixed G-invariant metric $g$, we have already noticed that every Killing field $\xi$ on $(M, g)$ is represented by a unique Hamiltonian $f_{\xi}$ with $\int_{M} f_{\xi} d \mu=0$, and that the Lie bracket on $\mathfrak{g}$ is thereby transformed into the Poisson bracket on $(M, \omega)$ :

$$
f_{[\xi, \eta]}=\left\{f_{\xi}, f_{\eta}\right\}=-\omega^{-1}\left(d f_{\xi}, d f_{\eta}\right) .
$$

Following Futaki and Mabuchi [25], we may therefore introduce a bilinear form $\mathbb{B}$ on the real Lie algebra $\mathfrak{g}$ by restricting the $L^{2}$ norm of $(M, g)$ to the space of these Hamiltonians:

$$
\mathbb{B}(\xi, \eta)=\int_{M} f_{\xi} f_{\eta} d \mu_{g}=\frac{1}{m !} \int_{M} f_{\xi} f_{\eta} \omega^{m} .
$$

Since a straightforward version of Moser stability shows that the Kähler forms of any two G-invariant metrics in a fixed Kähler class are Gequivariantly symplectomorphic, this inner product only depends on $\Omega$ and the maximal compact $\mathbf{G}<\mathbf{H}$, not on the representative metric $g$. Moreover, since any two maximal compacts are conjugate in $\mathbf{H}$, one can show [25] that the corresponding complex-bilinear form on $\mathfrak{g}_{\mathbb{C}}=\mathfrak{h} / \mathfrak{r}_{\mathfrak{u}}$ is actually independent of the choice of maximal compact $\mathbf{G}$.

Since $\mathbb{B}$ is positive-definite, and so defines an isomorphism $\mathfrak{g} \rightarrow \mathfrak{g}^{*}$, it also has a well-defined inverse which gives a positive-definite bilinear form

$$
\mathbb{B}^{-1}: \mathfrak{g}^{*} \times \mathfrak{g}^{*} \rightarrow \mathbb{R}
$$

on the Lie coalgebra of our maximal compact. On the other hand, assuming that $(M, J)$ is as in Proposition A.3, we have already seen that $\mathfrak{F}(\Omega) \in \mathfrak{g}^{*}$ for any Kähler class $\Omega$ on $M$. Thus, the number

$$
\|\mathfrak{F}(\Omega)\|^{2}:=\mathbb{B}^{-1}(\mathfrak{F}(\Omega), \mathfrak{F}(\Omega))
$$

is independent of choices, and so is an invariant of $(M, J, \Omega)$.

To see why this number has an important differential-geometric significance, let us first suppose that $g$ is a G-invariant Kähler metric with Kähler class $\Omega$, and let $\mathrm{p}$ be orthogonal projection in the real Hilbert space $L^{2}(M, g)$ to the subspace of normalized Hamiltonians representing the Lie algebra $\mathfrak{g}$ of Killing fields on $(M, g)$. Restricting equation (2.1) to $\mathfrak{g} \subset \mathfrak{h}$, one observes that $\mathfrak{F}(\Omega): \mathfrak{g} \rightarrow \mathbb{R}$ is exactly given by the $\mathbb{B}$-inner-product with the Killing 
field whose Hamiltonian is $-\mathrm{p}(s-\bar{s})$. We thus immediately have

$$
\int_{M}[\mathrm{p}(s-\bar{s})]^{2} d \mu_{g}=\|\mathfrak{F}(\Omega)\|^{2}
$$

and, since the projection $\mathrm{p}$ is norm-decreasing, it follows that

$$
\int_{M}(s-\bar{s})^{2} d \mu_{g} \geq\|\mathfrak{F}(\Omega)\|^{2}
$$

for any G-invariant Kähler metric with Kähler class $\Omega$. It is a remarkable fact, proved by Xiuxiong Chen [13], that inequality (2.3) actually holds even if $g$ is not assumed to be G-invariant. Moreover, equality holds in (2.3) if and only if $\nabla^{1,0} s$ is a holomorphic vector field, which is precisely the condition $[\mathbf{1 1}, \mathbf{1 2}]$ for $g$ to be an extremal Kähler metric.

The bilinear form $\mathbb{B}$ on $\mathfrak{g}$ is bi-invariant. In particular, the center $\mathfrak{z}$ of $\mathfrak{g}$ is $\mathbb{B}$-orthogonal to the semi-simple factor $[\mathfrak{g}, \mathfrak{g}]$ of $\mathfrak{g}$. Thus, a computation of $\|\mathfrak{F}(\Omega)\|^{2}$ does not require a complete knowledge of the bilinear form $\mathbb{B}$; only a knowledge of its restriction to $\mathfrak{z}$ is required. This observation allows us to prove the following:

Corollary 2.3. Let $(M, J)$ be as in Proposition 2.2, let $\mathbf{T}$ be a maximal torus in the complex automorphism group of $(M, J)$, and let $\mathfrak{t}$ denote the Lie algebra of $\mathbf{T}$. If $g$ is any $\mathbf{T}$-invariant Kähler metric with Kähler class $\Omega$, and if

$$
\mathbb{B}_{\mathbf{T}}: \mathfrak{t} \times \mathfrak{t} \rightarrow \mathbb{R}
$$

is the $g$-induced $L^{2}$-norm restricted to normalized Hamiltonians, then

$$
\|\mathfrak{F}(\Omega)\|^{2}=\mathbb{B}_{\mathbf{T}}^{-1}(\mathfrak{F}(\Omega), \mathfrak{F}(\Omega))
$$

where $\mathbb{B}_{\mathbf{T}}^{-1}$ denotes the inner product on $\mathfrak{t}^{*}$ induced by $\mathbb{B}_{\mathbf{T}}$.

Proof. Let $\mathbf{G}$ be a maximal compact subgroup of $\mathbf{H}$ containing $\mathbf{T}$. Then, by Proposition 2.2, the assertion certainly holds for any G-invariant Kähler metric $\tilde{g}$ in $\Omega$. However, by averaging, any $\mathbf{T}$-invariant Kähler metric with Kähler class $\Omega$ can be joined to $\tilde{g}$ by a path of such metrics, and is therefore $\mathbf{T}$-equivariantly symplectomorphic to $\tilde{g}$ by Moser stability. The claim therefore follows, since $\mathfrak{F}(\Omega) \in \mathfrak{t}^{*}$ is completely determined by $(M, J, \Omega)$, while $\mathbb{B}_{\mathbf{T}}$ is completely determined by the symplectic form and normalized Hamiltonians representing elements of $\mathfrak{t}$.

\section{Toric Manifolds}

We now specialize our discussion to the toric case. For clarity, our presentation will be self-contained, and will include idiosyncratic proofs of various standard facts about toric geometry. For more orthodox expositions of some of these fundamentals, the reader might do well to consult $[\mathbf{2 3}]$ and $[27]$.

We define a toric manifold to be a (connected) compact complex $\mathrm{m}$ manifold $\left(M^{2 m}, J\right)$ of Kähler type which has non-zero Euler characteristic 
and which is equipped a group of automorphisms generated by $m$ commuting, periodic, $J$-preserving real vector fields which are linearly independent in the space of vector fields on $M$. Thus, the relevant group of automorphisms $\mathbf{T}$ is required to be the image of the $m$-torus under some Lie group homomorphism $T^{m} \rightarrow \operatorname{Aut}(M, J)$ which induces an injection of Lie algebras. Notice that our definition implies that there must be a fixed point $p \in M$ of this $T^{m}$-action. Indeed, the fixed point set of any circle action on a smooth compact manifold is [32] a disjoint union of smooth compact manifolds with total Euler characteristic equal to the Euler characteristic of the ambient space; by induction on the number of circle factors, it follows that the fixed-point set of any torus action on $M$ therefore has total Euler characteristic $\chi(M) \neq 0$, and so, in particular, cannot be empty.

In light of this, let $p \in M$ be a fixed point of the given $T^{m}$-action on a toric manifold $\left(M^{2 m}, J\right)$, and, by averaging, also choose a Kähler metric $g$ on $M$ which is $T^{m}$-invariant. Then $T^{m}$ acts on $T_{p} M \cong \mathbb{C}^{m}$ in a manner preserving both $g$ and $J$, giving us a unitary representation $T^{m} \rightarrow \mathbf{U}(m)$. Since the action of $T^{m}$ on $T_{p} M$ completely determines the action on $M$ via the exponential map $T_{p} M \rightarrow M$ of $g$, and since, by hypothesis, the Lie algebra of $T^{m}$ injects into the vector fields on $M$, it follows that the above unitary representation gives rise to a faithful representation of $\mathbf{T}<\operatorname{Aut}(M, J)$. However, $\mathbf{U}(m)$ has rank $m$, so the image of $T^{m} \rightarrow \mathbf{U}(m)$ must be a maximal torus in $\mathbf{U}(m)$; thus, after a change of basis of $\mathbb{C}^{m}, \mathbf{T}$ may be identified with the standard maximal torus $\mathbf{U}(1) \times \cdots \times \mathbf{U}(1) \subset \mathbf{U}(m)$ consisting of diagonal matrices. In particular, $\mathbf{T}<\operatorname{Aut}(M, J)$ is intrinsically an $m$-torus, and has many free orbits. Since the origin in $\mathbb{C}^{m}$ is the only fixed point of the diagonal torus in $\mathbf{U}(m)$, it also follows that $p$ must be an isolated fixed point of $\mathbf{T}$. But since the same argument applies equally well to any other fixed point, this shows that the fixed-point set $M_{\mathbf{T}}$ of $\mathbf{T}$ is discrete, and therefore finite. In particular, $\chi(M)$ must equal the cardinality of $M_{\mathbf{T}}$, so the Euler characteristic of $M$ is necessarily positive.

The above arguments in particular show that the toric condition can be reformulated as follows: a toric $m$-manifold is a compact complex $m$-manifold $(M, J)$ of Kähler type, together with an $m$-torus $\mathbf{T} \subset \operatorname{Aut}(M, J)$ that has both a free orbit $\mathcal{Q}$ and a fixed point $p$. To check the equivalence, note that this reformulation implies that the Euler characteristic $\chi(M)$ is positive, because the fixed-point set $M_{\mathbf{T}}$ is necessarily finite, and by hypothesis is also non-empty.

Now let $(M, J, \mathbf{T})$ be a toric $m$-manifold, and let $\mathbf{j}: \mathcal{Q} \hookrightarrow M$ be the inclusion of a free $\mathbf{T}$-orbit. Since $\mathbf{T}$ also has a fixed-point $p$, and since any two $\mathbf{T}$-orbits are homotopic, it follows that $\mathfrak{j}$ is homotopic to a constant map. Consequently, the induced homomorphism $\mathfrak{j}^{*}: H^{k}(M) \rightarrow H^{k}(\mathcal{Q})$ must be the zero map in all dimensions $k>0$. However, the restriction of the Kähler form $\omega=g(J \cdot, \cdot)$ to $\mathcal{Q} \approx \mathbf{T}$ is an invariant 2 -form on $\mathbf{T} \approx T^{m}$. Since every deRham class on $T^{m}$ contains a unique invariant form, and since $\mathfrak{j}^{*}[\omega]=0 \in H^{2}\left(T^{m}, \mathbb{R}\right)$, it follows that $\mathfrak{j}^{*} \omega$ must vanish identically. Thus $\mathcal{Q}$ is 
a Lagrangian submanifold, which is to say that $T \mathcal{Q}$ is everywhere orthogonal to $J(T \mathcal{Q})$. In particular, if $\xi_{1}, \ldots, \xi_{m}$ are the generators of the $\mathbf{T}$-action, the corresponding holomorphic vector fields $\Xi_{j}=-\left(J \xi_{j}+i \xi_{j}\right) / 2$ span $T^{1,0} M$ in a neighborhood of $\mathcal{Q}$. Integrating the flows of the commuting vector fields $\xi_{j}$ and $J \xi_{j}$, we thus obtain a holomorphic action of the complexified torus $\left(\mathbb{C}^{\times}\right)^{m}$ which has both a fixed point and an open orbit $\mathcal{U}$.

In particular, $(M, J)$ carries $m$ holomorphic vector fields $\Xi_{1}, \ldots, \Xi_{m}$ which vanish at $p$, but which nonetheless $\operatorname{span} T^{1,0}(M)$ at a generic point. It follows that $M$ cannot carry a non-trivial holomorphic $k$-form $\alpha \in H^{k, 0}(M)$ for any $k>0$, since, for any choice of $j_{1}, \ldots, j_{k}$, the "component" functions $\alpha\left(\Xi_{j_{1}}, \ldots, \Xi_{j_{k}}\right)$ would be holomorphic, and hence constant, and yet would have to vanish at the fixed point $p$. In particular, we may invoke Kodaira's observation [33] that any Kähler manifold with $H^{2,0}=0$ admits Hodge metrics, and so is projective. This gives us the following result:

LEMMA 3.1. Any toric manifold $M$ is projective algebraic, and satisfies $H^{k, 0}(M)=0$ for all $k>0$.

In particular, the identity component $\mathbf{H}=\operatorname{Aut}^{0}(M, J)$ of the automorphism group of our toric $m$-manifold is linear algebraic. Let $\mathbf{T}<\mathbf{H}$ be the $m$-torus associated with the toric structure of $(M, J)$. Using the Chevalley decomposition, we can then choose a maximal compact subgroup $\mathbf{G}<\mathbf{H}$ containing T. Also choose a G-invariant Kähler metric $g$ on $M$ and a fixed point $p$ of $\mathbf{T}$. We will now study the centralizer $Z(\mathbf{T})<\mathbf{G}$, consisting of elements of $\mathbf{G}$ that commute with all elements of $\mathbf{T}$. Observe that

$$
a \in Z(\mathbf{T}), b \in \mathbf{T} \Longrightarrow 6(a(p))=a(b(p))=a(p),
$$

so that $Z(\mathbf{T})$ acts by permutation on the finite set $M_{\mathbf{T}}$ of fixed points. In particular, the identity component $Z^{0}(\mathbf{T})$ of $Z(\mathbf{T})$ must send $p$ to itself. Once more invoking the exponential map of $g$, we thus obtain a faithful unitary representation of $Z^{0}(\mathbf{T})$ by considering its induced action on $T_{p} M \cong \mathbb{C}^{m}$. However, the image of $Z^{0}(\mathbf{T})$ in $\mathbf{U}(m)$ must then be a subgroup of the centralizer of the diagonal torus $\mathbf{U}(1) \times \cdots \times \mathbf{U}(1)$ in $\mathbf{U}(m)$. But since the latter centralizer is just the diagonal torus itself, we conclude that $Z^{0}(\mathbf{T})=\mathbf{T}$. It follows that $\mathbf{T}$ is a maximal torus in $\mathbf{G}$, and hence also in $\mathbf{H}=\mathbf{G}_{\mathbb{C}} \ltimes \mathbf{R}_{\mathbf{u}}$ :

Lemma 3.2. Let $\left(M^{2 m}, J\right)$ be a toric manifold, and let $\mathbf{T}<\operatorname{Aut}(M, J)$ be the associated $m$-torus. Then $\mathbf{T}$ is a maximal torus in $\operatorname{Aut}(M, J)$.

Combining this result with Lemma 3.1 and Proposition 2.2, we can thus generalize [46, Theorem 1.9] to irrational Kähler classes:

Proposition 3.3. Let $\left(M^{2 m}, J\right)$ be a toric manifold, let $\mathbf{T}$ be the given $m$-torus in its automorphism group, and let $\mathfrak{t}$ be the Lie algebra of $\mathbf{T}$. Then, for any Kähler class $\Omega$ on $M$, the Futaki invariant $\mathfrak{F}(\Omega)$ naturally belongs to $\mathfrak{t}^{*}$. In particular, $\mathfrak{F}(\Omega)$ is completely determined by its restriction to $\mathfrak{t}$. 
However, we will not simply need to know where $\mathfrak{F}(\Omega)$ lives; our goal will require us to calculate its norm with respect to the relevant bilinear form. Fortunately, Lemma 3.2 and Corollary 2.3 together imply the following result:

Proposition 3.4. Let $\left(M^{2 m}, J\right)$ be a toric manifold, let $\mathbf{T}$ be the given $m$-torus in its automorphism group, and let $\mathfrak{t}$ be the Lie algebra of $\mathbf{T}$. If $g$ is any $\mathbf{T}$-invariant Kähler metric with Kähler class $\Omega$, and if

$$
\mathbb{B}_{\mathbf{T}}: \mathfrak{t} \times \mathfrak{t} \rightarrow \mathbb{R}
$$

is the $g$-induced $L^{2}$-norm restricted to normalized Hamiltonians, then

$$
\|\mathfrak{F}(\Omega)\|^{2}=\mathbb{B}_{\mathbf{T}}^{-1}(\mathfrak{F}(\Omega), \mathfrak{F}(\Omega))
$$

where $\mathbb{B}_{\mathbf{T}}^{-1}$ denotes the inner product on $\mathfrak{t}^{*}$ induced by $\mathbb{B}_{\mathbf{T}}$.

Of course, Lemma 3.1 has many other interesting applications. For example, by Hodge symmetry, it implies the Todd genus is given by

$$
\chi(M, \mathcal{O})=\sum_{k}(-1)^{k} h^{0, k}(M)=1
$$

for any toric manifold $M$. Since the same argument could also be applied to any finite covering of $M$, whereas $\chi(M, \mathcal{O})$ is multiplicative under coverings, one immediately sees that $M$ cannot have non-trivial finite covering spaces. In particular, this implies that $H_{1}(M, \mathbb{Z})=0$.

However, one can easily do much better. Choose a T-invariant Kähler metric $g$ with Kähler form $\omega$. Because $b_{1}(M)=0$ by Lemma 3.1, the symplectic vector fields $\xi_{1}, \ldots, \xi_{m}$ must then have Hamiltonians, so that $\xi_{j}=$ $J \nabla f_{j}$ for suitable functions $f_{1}, \ldots, f_{m}$. Let $a_{1}, \ldots, a_{m}$ be real numbers which are linearly independent over $\mathbb{Q}$, and let $f=\sum_{j} a_{j} f_{j}$. The corresponding symplectic vector field $\xi=\sum_{j} a_{j} \xi_{j}$ is thus a Killing field for $g$, and its flow is dense in the torus $\mathbf{T}<\operatorname{Aut}(M, J)$. Consequently, $\xi$ vanishes only at the fixed points of $\mathbf{T}$. Since $\xi$ is Killing, with only isolated zeroes, it then follows that $\nabla \xi$ is non-degenerate at each fixed point $p$ of $\mathbf{T}$, in the sense that it defines an isomorphism $T_{p} \rightarrow T_{p}$. Since $\nabla_{a} \nabla_{b} f=\omega_{b c} \nabla_{a} \xi^{c}$, this implies that the Hessian of $f$ is non-degenerate at each zero of $d f$; that is, $f$ is a Morse function on $M$. However, since $\xi$ is the real part of a holomorphic vector field, $\bar{\partial} \partial^{\#} f=0$, and this is equivalent to saying that the Riemannian Hessian $\nabla \nabla f$ is everywhere $J$-invariant. Since the Riemannian Hessian coincides with the naïve Hessian at a critical point, this shows that every critical point of $f$ must have even index. It follows [44] that $M$ is homotopy equivalent to a $C W$ complex consisting entirely of even-dimensional cells. In particular, we obtain the following:

Lemma 3.5. Any toric manifold is simply connected, and has trivial homology in all odd dimensions. 
Finally, notice that Lemma 3.1 implies that the canonical line bundle $K=\Lambda^{m, 0}$ of a toric $m$-manifold has no non-trivial holomorphic sections. However, essentially the same argument also shows that positive powers $K^{\ell}$ cannot have non-trivial holomorphic sections either, since the pairing of such a section with $\left(\Xi_{1} \wedge \cdots \wedge \Xi_{m}\right)^{\otimes \ell}$ would again result in a constant function which would have to vanish at $p$. Thus, all the plurigenera $p_{\ell}=h^{0}\left(\mathcal{O}\left(K^{\ell}\right)\right)$ of any toric manifold must vanish. In other words:

Lemma 3.6. Any toric manifold has Kodaira dimension $-\infty$.

\section{The Virtual Action}

As previously discussed in connection with (2.3), a theorem of Chen [13] says that any Kähler metric $g$ on a compact complex manifold $M$ satisfies

$$
\int_{M}(s-\bar{s})^{2} d \mu_{g} \geq\|\mathfrak{F}(\Omega)\|^{2},
$$

where $\Omega=[\omega]$ is the Kähler class of $g$; moreover, equality holds iff $g$ is an extremal Kähler metric. On the other hand,

$$
\int_{M} s^{2} d \mu_{g}=\int_{M}(s-\bar{s})^{2} d \mu_{g}+\int_{M} \bar{s}^{2} d \mu_{g}
$$

as may be seen by applying the Pythagorean theorem to $L^{2}$-norms. Since $s$ is the trace of the Ricci tensor with respect to the metric, and because the Ricci form is essentially the curvature of the canonical line bundle, we also know that

$$
\int_{M} s d \mu=\frac{4 \pi c_{1} \cdot \Omega^{m-1}}{(m-1) !}
$$

in complex dimension $m$; meanwhile, the volume of an $m$-dimensional Kähler $m$-manifold is just given by

$$
\int_{M} d \mu=\frac{\Omega^{m}}{m !}
$$

Hence

$$
\int_{M} \bar{s}^{2} d \mu=\frac{\left(\int_{M} s d \mu\right)^{2}}{\int_{M} d \mu}=\frac{16 \pi^{2} m}{(m-1) !} \frac{\left(c_{1} \cdot \Omega^{m-1}\right)^{2}}{\Omega^{m}}
$$

and (4.1) thus implies that

$$
\int_{M} s^{2} d \mu_{g} \geq \frac{16 \pi^{2} m}{(m-1) !} \frac{\left(c_{1} \cdot \Omega^{m-1}\right)^{2}}{\Omega^{m}}+\|\mathfrak{F}(\Omega)\|^{2}
$$

with equality iff $g$ is an extremal Kähler metric on $\left(M^{2 m}, J\right)$.

Now specializing to the case of complex dimension $m=2$, we have

$$
\int_{M} s^{2} d \mu_{g} \geq 32 \pi^{2} \frac{\left(c_{1} \cdot \Omega\right)^{2}}{\Omega^{2}}+\|\mathfrak{F}(\Omega)\|^{2}
$$


for any Kähler metric $g$ with Kähler class $[\omega]=\Omega$ on a compact complex surface $\left(M^{4}, J\right)$. In other words, if we define a function on the Kähler cone by

then

$$
\mathcal{A}(\Omega):=\frac{\left(c_{1} \cdot \Omega\right)^{2}}{\Omega^{2}}+\frac{1}{32 \pi^{2}}\|\mathfrak{F}(\Omega)\|^{2}
$$

$$
\frac{1}{32 \pi^{2}} \int_{M} s_{g}^{2} d \mu_{g} \geq \mathcal{A}(\Omega)
$$

for any Kähler metric $g$ with Kähler class $\Omega$, with equality iff $g$ is an extremal Kähler metric. The function $\mathcal{A}(\Omega)$ will be called the virtual action. Our normalization has been chosen so that $\mathcal{A}(\Omega) \geq c_{1}^{2}(M)$, with equality iff the Futaki invariant vanishes and $\Omega$ is a multiple of $c_{1}$. (Incidentally, the latter occurs iff $\Omega$ is the Kähler class of a Kähler-Einstein metric on $\left(M^{4}, J\right)$ $[4,47,52,54]$.) The fact that the virtual action $\mathcal{A}(\Omega)$ is homogeneous of degree 0 in $\Omega$ corresponds to the fact that the Calabi energy $\mathcal{C}(g)$ is scaleinvariant in real dimension four.

In complex dimension $m=2$, one important reason for studying the Calabi energy $\mathcal{C}$ is the manner in which (1.2) relates it to the Weyl functional

$$
\mathcal{W}(g)=\int_{M}|W|_{g}^{2} d \mu_{g}
$$

where the Weyl curvature $W$ is the conformally invariant piece of the curvature tensor. It is easy to check that $\mathcal{W}$ is also conformally invariant, and may therefore be considered as a functional on the space of conformal classes of Riemannian metrics. Critical points of the Weyl functional are characterized $[\mathbf{5}, \mathbf{9}]$ by the vanishing of the Bach tensor

$$
B_{a b}:=\left(\nabla^{c} \nabla^{d}+\frac{1}{2} r^{c d}\right) W_{a c b d}
$$

and so are said to be Bach-flat; obviously, this is a conformally invariant condition. The Bianchi identities immediately imply that any Einstein metric on a 4-manifold is Bach-flat, and it therefore follows that any conformally Einstein metric is Bach-flat, too. The converse, however, is false; for example, self-dual and anti-self-dual metrics are also Bach-flat, and such metrics exist on many compact 4 -manifolds $[\mathbf{3 4}, \mathbf{3 5}, \mathbf{5 1}]$ that do not admit Einstein metrics.

When the Weyl functional $\mathcal{W}$ is restricted to the space of Kähler metrics, equation (1.2) shows that it becomes equivalent to the Calabi energy $\mathcal{C}$. Nonetheless, the following result [15] may come as something of a surprise:

Proposition 4.1. Let $g$ be a Kähler metric on a compact complex surface. Then $g$ is Bach-flat if and only if

- $g$ is an extremal Kähler metric, and

- its Kähler class $\Omega$ is a critical point of the virtual action $\mathcal{A}$.

This gives rise to a remarkable method of constructing Einstein metrics, courtesy of a beautiful result of Derdziński [19, Proposition 4]: 
Proposition 4.2. If the scalar curvature s of a Bach-flat Kähler metric $g$ on a complex surface $\left(M^{4}, J\right)$ is not identically zero, then the conformally related metric $h=s^{-2} g$ is Einstein on the open set $s \neq 0$ where it is defined.

\section{Toric Surfaces}

We will now prove Theorem A by computing the virtual action $\mathcal{A}(\Omega)$ for any Kähler class on a toric surface. An important intermediate step in this process involves an explicit computation of the Futaki invariant $\mathfrak{F}(\Omega)$. Up to a universal constant, our answer agrees with that of various other authors $[\mathbf{2 1}, \mathbf{2 5}, \mathbf{4 1}, \mathbf{4 9}]$, but determining the correct constant is crucial for our purposes. For this reason, our first proof will be based on the author's formula [34] for the scalar curvature of a Kähler surface with isometric $S^{1}$ action.

By a toric surface, we mean a toric manifold $(M, J, \mathbf{T})$ of complex dimension two. This is equivalent ${ }^{1}$ to saying that $\left(M^{4}, J\right)$ is a simply connected compact complex surface equipped with a 2 -torus $\mathbf{T}<\operatorname{Aut}(M, J)$. By Castelnuovo's criterion $[\mathbf{7 , 2 6}$, Lemma 3.1 and Lemma 3.6, any toric surface $(M, J)$ can be obtained from either $\mathbb{C P}_{2}$ or a Hirzebruch surface by blowing up points. Indeed, since the holomorphic vector fields generating the torus action on $M$ automatically descend to the minimal model, the toric structure of $(M, J)$ can be obtained from a toric structure on $\mathbb{C P}_{2}$ or a Hirzebruch surface by iteratively blowing up fixed points of the torus action. For more direct proofs, using the toric machinery of fans or moment polytopes, see $[\mathbf{2 3}, \mathbf{2 7}]$.

Let $\left(M^{4}, J, \mathbf{T}, \Omega\right)$ now be a toric surface with fixed Kähler class. By averaging, we can then find a $\mathbf{T}$-invariant Kähler metric $g$ on $(M, J)$ with Kähler form $\omega \in \Omega$. Choose an isomorphism $\mathbf{T} \cong \mathbb{R}^{2} / \mathbb{Z}^{2}$, and denote the corresponding generating vector fields of period 1 by $\xi_{1}$ and $\xi_{2}$. Since $b_{1}(M)=0$, there are Hamiltonian functions $x_{1}$ and $x_{2}$ on $M$ with $\xi_{j}=$ $J \operatorname{grad} x_{j}, j=1,2$. This makes $(M, \omega)$ into a Hamiltonian $T^{2}$-space in the sense of [27]. In particular, the image of $M$ under $\vec{x}=\left(x_{1}, x_{2}\right)$ is $[\mathbf{3}, \mathbf{2 8}]$ a convex polygon $P \subset \mathbb{R}^{2}$ whose area is exactly the volume of $(M, g)$. The map $\vec{x}: M \rightarrow \mathbb{R}^{2}$ is called the moment map, and its image $P=\vec{x}(M)$ will be called the moment polygon. Of course, since we have not insisted that the Hamiltonians $x_{k}$ have integral zero, our moment map is only determined up to translations of $\mathbb{R}^{2}$. Modulo this ambiguity, however, the moment polygon is uniquely determined by $(M, \omega, \mathbf{T})$, together with the chosen basis $\left(\xi_{1}, \xi_{2}\right)$ for the Lie algebra $\mathfrak{t}$ of of $\mathbf{T}$. Moreover, since a straightforward Moserstability argument shows that any two $\mathbf{T}$-invariant Kähler forms in $\Omega$ are $\mathbf{T}$ equivariantly symplectomorphic, the moment polygon really only depends on $\left(M, J, \Omega,\left(\xi_{1}, \xi_{2}\right)\right)$. However, outer automorphisms of $\mathbf{T}$ can be used to

\footnotetext{
${ }^{1}$ In one direction, this equivalence follows because any simply connected compact complex surface is of Kähler type $[\mathbf{1 0}, \mathbf{5 0}]$ and has positive Euler characteristic. On the other hand, the converse follows from Lemma 3.5.
} 
alter $\left(\xi_{1}, \xi_{2}\right)$ by an $\mathbf{S L}(2, \mathbb{Z})$ transformation, and this in turn changes the moment polygon by an $\mathbf{S L}(2, \mathbb{Z})$ transformation of $\mathbb{R}^{2}$. Moreover, since the vertices of $P$ correspond to the fixed points of $\mathbf{T}$, and because the action of $\mathbf{T}$ on the tangent space of any fixed point can be identified with that of the diagonal torus $\mathbf{U}(1) \times \mathbf{U}(1) \subset \mathbf{U}(2)$, a neighborhood of any corner of $P$ can be transformed into a neighborhood of the origin in the positive quadrant of $\mathbb{R}^{2}$ by an element of $\mathbf{S L}(2, \mathbb{Z})$ and a translation $[\mathbf{1 8}]$. Polygons with the latter property are said to be Delzant, and any Delzant polygon arises from a uniquely determined toric surface, equipped with a uniquely determined Kähler class [27].

We now introduce a measure $d \lambda$ on the boundary $\partial P$ of our moment polygon. To do this, first notice that each edge of $P$ is the image of a rational curve $C_{\imath} \cong \mathbb{C P}_{1}$ in $(M, J)$ which is fixed by an $S^{1}$ subgroup of $T^{2}$, and hence by a $\mathbb{C}^{\times}$subgroup of the complexified torus $\mathbb{C}^{\times} \times \mathbb{C}^{\times}$. We then define the measure $d \lambda$ along the edge $\ell_{\imath}=\vec{x}\left(C_{\imath}\right)$ to be the push-forward, via $\vec{x}$, of the smooth area measure on $C_{\imath}$ given by the restriction of the Kähler form $\omega$. Since a rational linear combination of the $x_{k}$ is a Hamiltonian for rotation of $C_{\imath}$ about two fixed points, $d \lambda$ is a constant times 1-dimensional Lebesgue measure on the line segment $\ell_{2}$, with total length

$$
\int_{\ell_{\imath}} d \lambda=\int_{C_{\imath}} \omega=: \mathcal{A}_{\imath}
$$

equal to the area of corresponding holomorphic curve in $M$. Here the index $\imath$ is understood to run over the edges of $\partial P$.

When an edge is parallel to either axis, $d \lambda$ just becomes standard Euclidean length measure. More generally, on an arbitrary edge, it must coincide with the pull-back of Euclidean length via any $\mathbf{S L}(2, \mathbb{Z})$ transformation which sends the edge to a segment parallel to an axis. Because $P$ is a Delzant polygon, this contains enough information to completely determine $d \lambda$, and leads to a consistent definition of the measure because the stabilizer

$$
\left\{ \pm\left(\begin{array}{cc}
1 & k \\
0 & 1
\end{array}\right) \mid k \in \mathbb{Z}\right\}
$$

of the $x_{1}$-axis in $\mathbf{S L}(2, \mathbb{Z})$ preserves Euclidean length on this axis. However, the Euclidean algorithm of elementary number theory implies that every pair $(p, q)$ of relatively prime non-zero integers belongs to the $\mathbf{S L}(2, \mathbb{Z})$-orbit of $(1,0)$. One can therefore compute edge-lengths with respect to $d \lambda$ by means of the following recipe: Given an edge of $P$ which is not parallel to either axis, its slope $m$ is a non-zero rational number, and so can be expressed in lowest terms as $m=q / p$, where $p$ and $q$ are relatively prime non-zero integers. The displacement vector $\vec{v}$ representing the difference between the two endpoints of the edge can thus be written as $\vec{v}=(u p, u q)$ for some $u \in \mathbb{R}-\{0\}$. The length of the edge with respect to $d \lambda$ then equals $|u|$.

We can now associate two different barycenters with our moment polygon. First, there is the barycenter $\overline{\vec{x}}=\left(\bar{x}_{1}, \bar{x}_{2}\right)$ of the interior of $P$, as defined 
by

$$
\bar{x}_{k}=\int_{P} x_{k} d a=\frac{\int_{P} x_{k} d a}{\int_{P} d a}
$$

where $d a$ is standard 2-dimensional Lebesgue measure in $\mathbb{R}^{2}$. Second, there is the barycenter $\langle\vec{x}\rangle=\left(\left\langle x_{1}\right\rangle,\left\langle x_{2}\right\rangle\right)$ of the perimeter $\partial P$, defined by

$$
\left\langle x_{k}\right\rangle=\int_{\partial P} x_{k} d \lambda=\frac{\int_{\partial P} x_{k} d \lambda}{\int_{\partial P} d \lambda}
$$

These two barycenters certainly need not coincide in general. It is therefore natural to consider the displacement vector

$$
\overrightarrow{\mathfrak{D}}=\langle\vec{x}\rangle-\overline{\vec{x}}
$$

that measures their separation. Notice that $\overrightarrow{\mathfrak{D}}$ is translation invariant - it is unchanged if we alter the Hamiltonians $\left(x_{1}, x_{2}\right)$ by adding constants.

Next, we introduce the moment-of-inertia matrix $\Pi$ of $P$, which encodes the moment of inertia of the polygon about an arbitrary axis in $\mathbb{R}^{2}$ passing through its barycenter $\overrightarrow{\vec{x}}$. Thus $\Pi$ is the positive-definite symmetric $2 \times 2$ matrix with entries given by

$$
\Pi_{j k}=\int_{P}\left(x_{j}-\bar{x}_{j}\right)\left(x_{k}-\bar{x}_{k}\right) d a
$$

where $d a$ once again denotes the usual Euclidean area form on the interior of $P$, and exactly equals the push-forward of the metric volume measure on $M$. For our purposes, it is important to notice that $\Pi$ is always an invertible matrix.

Finally, let $|\partial P|=\int_{\partial P} d \lambda=\sum_{\imath} \mathcal{A}_{\imath}$ denote the perimeter of the moment polygon with respect to the measure $d \lambda$ introduced above, and let $|P|=$ $\int_{P} d a$ denote the area of its interior in the usual sense. With these notational conventions, we are now ready to state the main result of this section:

TheOREm 5.1. If $(M, J, \Omega)$ is any toric surface with fixed Kähler class, then

$$
\mathcal{A}(\Omega)=\frac{|\partial P|^{2}}{2}\left(\frac{1}{|P|}+\overrightarrow{\mathfrak{D}} \cdot \Pi^{-1} \overrightarrow{\mathfrak{D}}\right)
$$

where $P$ is the moment polygon determined by the given $T^{2}$-action.

The proof of Theorem 5.1 crucially depends on a computation of the Futaki invariant, which, we recall, is a character on the Lie algebra of holomorphic vector fields. Let us therefore consider the holomorphic vector fields $\Xi_{k}=\nabla^{1,0} x_{k}$ whose holomorphy potentials are the Hamiltonians of the periodic Killing fields $\xi_{k}$. These are explicitly given by

$$
\Xi_{k}=-\frac{1}{2}\left(J \xi_{k}+i \xi_{k}\right)
$$


Proposition 5.2. Suppose that $(M, J, \Omega)$ is a toric surface with fixed Kähler class, and let $\Xi_{k}$ be the generators of the associated complex torus action, normalized as above. Let

$$
\mathfrak{F}_{k}:=\mathfrak{F}\left(\Xi_{k}, \Omega\right)
$$

be the corresponding components of the Futaki invariant of $(M, J, \Omega)$. Then the vector $\overrightarrow{\mathfrak{F}}=\left(\mathfrak{F}_{1}, \mathfrak{F}_{2}\right)$ is explicitly given by

$$
\overrightarrow{\mathfrak{F}}=-4 \pi|\partial P| \overrightarrow{\mathfrak{D}}
$$

where $|\partial P|$ again denotes the weighted perimeter of the moment polygon $P$, and $\overrightarrow{\mathfrak{D}}$ is again the vector joining the barycenters of the interior and weighted boundary of $P$.

Proof. More explicitly, the assertion is that

$$
\mathfrak{F}_{k}=-4 \pi \sum_{\imath}\left(\left\langle x_{k}\right\rangle_{\imath}-\bar{x}_{k}\right) \mathcal{A}_{\imath}
$$

where $\bar{x}_{k}$ is once again the $k^{\text {th }}$ coordinate of the barycenter of the interior of the moment polygon $P,\left\langle x_{k}\right\rangle_{\imath}$ is the $k^{\text {th }}$ coordinate of the center of the $\imath^{\text {th }}$ edge of $P$, and $\mathcal{A}_{\imath}$ is the weighted length of $\imath^{\text {th }}$ edge.

We will now prove (5.2) using a method $[\mathbf{3 1}, 40]$ which is broadly applicable to $\mathbb{C}^{\times}$-actions, but which nicely simplifies in the toric case. We thus make a choice of $k=1$ or 2 , and set $\Xi=\Xi_{k}, \xi=\xi_{k}$, and $x=x_{k}$ for this choice of $k$. In order to facilitate comparison with $[\mathbf{3 1}, \mathbf{3 4}, \mathbf{4 0}]$, set $\eta=\xi / 2 \pi$, so that $\eta$ is a symplectic vector field of period $2 \pi$, with Hamiltonian $t=x / 2 \pi$. Let $\Sigma=M / / \mathbb{C}^{\times}$be the stable quotient of $(M, J)$ by the action generated by $\Xi$, and observe that the following interesting special properties hold in our toric setting:

- the stable quotient $\Sigma$ has genus 0 ; and

- all the isolated $\mathbb{C}^{\times}$fixed points project to just two points $q_{1}, q_{2} \in \Sigma$.

Let $\mathbf{a}, \mathbf{b} \in \mathbb{R}$, respectively, denote the minimum and maximum of the Hamiltonian $t$, so that $t(M)=[\mathbf{a}, \mathbf{b}]$. If $t^{-1}(\{\mathbf{a}\})$ or $t^{-1}(\{\mathbf{b}\})$ is an isolated fixed point, blow up $M$ there to obtain $\hat{M}$, and pull the metric $g$ back to $\hat{M}$ as a degenerate metric; otherwise, let $\hat{M}=M$. We then have a holomorphic quotient map $\varpi: \hat{M} \rightarrow \Sigma$. Let $C_{+}$and $C_{-}$be the holormorphic curves in $\hat{M}$ given by $t^{-1}(\mathbf{b})$ and $t^{-1}(\mathbf{a})$, respectively. Except when they are just artifacts produced by blowing up, the curves $C_{ \pm}$number among the rational curves $C_{\imath}$ which project to the sides of the moment polygon $P$; the others, after proper transform if necessary, form a sub-collection $\left\{E_{\jmath}\right\} \subset\left\{C_{\imath}\right\}$ characterized by $\varpi^{-1}\left(\left\{q_{1}, q_{2}\right\}\right)=\cup_{\jmath} E_{\jmath}$ for a preferred pair of distinct points $q_{1}, q_{2} \in \Sigma$. Each $E_{\jmath}$ is the closure of a $\mathbb{C}^{\times}$-orbit, and we will let $m_{\jmath} \in \mathbb{Z}^{+}$denote the order of the isotropy of $\mathbb{C}^{\times}$acting on the relevant orbit. Also let $t_{\jmath}^{-}$and $t_{\jmath}^{+}$denote the minimum and maximum of $t$ on $E_{\jmath}$, so that $t\left(E_{\jmath}\right)=\left[t_{\jmath}^{-}, t_{\jmath}^{+}\right]$, and observe that

$$
\langle t\rangle_{\jmath}:=\left(t_{\jmath}^{-}+t_{\jmath}^{+}\right) / 2
$$


coincides with the average value of $t$ on $E_{\jmath}$ with respect to $g$-area measure.

Let us now define $\wp: \hat{M} \rightarrow \Sigma \times[\mathbf{a}, \mathbf{b}]$ to be the map $\varpi \times t$. If $p_{1}, \ldots, p_{m}$ are the images in $\Sigma \times(\mathbf{a}, \mathbf{b})$ of the isolated fixed points, and if

$$
X=[\Sigma \times(\mathbf{a}, \mathbf{b})]-\left\{p_{1}, \ldots, p_{m}\right\},
$$

then the open dense set $Y=\wp^{-1}(X) \subset \hat{M}$ map be viewed as an orbifold $S^{1}$-principal bundle over $X$, and comes equipped with a unique connection 1 -form $\theta$ whose kernel is $g$-orthogonal to $\eta$ and which satisfies $\theta(\eta)=1$. We may now express the given Kähler metric $g$ as

$$
g=w \check{g}(t)+w d t^{\otimes 2}+w^{-1} \theta^{\otimes 2},
$$

for a positive functions $w>0$ on $X$ and a family orbifold metrics $\check{g}(t)$ on $\Sigma$.

Because $g, w$ and $d t$ are geometrically defined, $\check{g}(t)$ is an invariantly defined, $t$-dependent orbifold Kähler metric on $\Sigma$ for all regular values of $t$; moreover, it is a smooth well-defined tensor field on all of $\left(\Sigma-\left\{q_{1}, q_{2}\right\}\right) \times$ $(\mathbf{a}, \mathbf{b})$. Now notice that the Kähler quotient of $M$ associated with a regular value of the Hamiltonian is manifestly $(\Sigma, w \check{g}(t))$, and must therefore tend to the restriction of $g$ to $C_{ \pm}$as $t \rightarrow \mathbf{a}$ or $\mathbf{b}$. On the other hand, $w^{-1}=g(\eta, \eta)$ by construction, and since $\eta$ is a Killing field of period $2 \pi$ and Hamiltonian $t$, we have $g(\eta, \eta)=2|t-\mathbf{a}|+O\left(|t-\mathbf{a}|^{2}\right)$ near $t=\mathbf{a}$, and similarly near $t=\mathbf{b}$. Thus $[\mathbf{3 1}, \mathbf{4 0}]$, letting $\check{\omega}(t)$ be the Kähler form of $\check{g}(t)$, we have

$$
\begin{aligned}
\left.\check{\omega}\right|_{t=\mathbf{a}}=\left.\check{\omega}\right|_{t=\mathbf{b}} & =0 \\
\left.\frac{d}{d t} \check{\omega}\right|_{t=\mathbf{a}} & =\left.2 \omega\right|_{C^{-}} \\
\left.\frac{d}{d t} \check{\omega}\right|_{t=\mathbf{b}} & =-\left.2 \omega\right|_{C^{+}} .
\end{aligned}
$$

More surprisingly, the calculations underlying the hyperbolic ansatz of [34] show [40, equation (3.16)] that the scalar curvature density of $g$ may be globally expressed on $Y \subset M$ as

$$
s d \mu=\left[2 \check{\rho}-\frac{d^{2}}{d t^{2}} \check{\omega}\right] \wedge d t \wedge \theta
$$

where $\check{\rho}(t)$ is the Ricci form of $\check{g}(t)$. However, for regular values of $t \in(\mathbf{a}, \mathbf{b})$, the Gauss-Bonnet formula for orbifolds tells us that

$$
\begin{aligned}
\frac{1}{2 \pi} \int_{\Sigma} \check{\rho}(t) & =\chi(\Sigma)-\sum_{\jmath} \delta_{\jmath}(t)\left(1-\frac{1}{m_{\jmath}}\right) \\
& =\chi\left(S^{2}\right)-2+\sum_{\jmath} \frac{1}{m_{\jmath}} \delta_{\jmath}(t) \\
& =\sum_{\jmath} \frac{1}{m_{\jmath}} \delta_{\jmath}(t)
\end{aligned}
$$


where we have introduced the characteristic function

$$
\delta_{\jmath}(t)= \begin{cases}1 & t_{\jmath}^{-}<t<t_{\jmath}^{+} \\ 0 & \text { otherwise }\end{cases}
$$

of $\left(t_{\jmath}^{-}, t_{\jmath}^{+}\right)$in order to keep track of which two curves $E_{\jmath}$ meet a given regular level-set of the Hamiltonian function $t$.

Now the Futaki invariant is defined in terms of the $L^{2}$ inner product of the scalar curvature $s$ of $g$ with normalized holomorphy potentials. It is therefore pertinent to observe that

$$
\begin{aligned}
\int_{M} t s d \mu & =\int_{Y} t s d \mu \\
& =\int_{Y} t\left[2 \check{\rho}-\frac{d^{2}}{d t^{2}} \check{\omega}\right] \wedge d t \wedge \theta \\
& =4 \pi \int_{\mathbf{a}}^{\mathbf{b}} t\left[\int_{\Sigma} \check{\rho}\right] d t-2 \pi \int_{\Sigma}\left[\int_{\mathbf{a}}^{\mathbf{b}} t \frac{d^{2}}{d t^{2}} \check{\omega}\right] d t \\
& =4 \pi \int_{\mathbf{a}}^{\mathbf{b}} 2 \pi\left[\sum_{\jmath} \frac{1}{m_{\jmath}} \delta_{\jmath}(t)\right] t d t-2 \pi \int_{\Sigma}\left(\left[t \frac{d}{d t} \check{\omega}\right]_{\mathbf{a}}^{\mathbf{b}}-\int_{\mathbf{a}}^{\mathbf{b}} \frac{d \check{\omega}}{d t} d t\right) \\
& =4 \pi \sum_{\jmath} \frac{2 \pi}{m_{\jmath}} \int_{t_{\jmath}^{-}}^{t_{\jmath}^{+}} t d t-2 \pi \int_{\Sigma}\left(-\left.2 \mathbf{b} \omega\right|_{t=\mathbf{b}}-\left.2 \mathbf{a} \omega\right|_{t=\mathbf{a}}-[\check{\omega}]_{\mathbf{a}}^{\mathbf{b}}\right) \\
& =4 \pi \sum_{\jmath} \frac{2 \pi\left(t_{\jmath}^{+}-t_{\jmath}^{-}\right)}{m_{\jmath}} \frac{t_{\jmath}^{+}+t_{\jmath}^{-}}{2}+4 \pi\left(\mathbf{a}[\omega] \cdot C^{-}+\mathbf{b}[\omega] \cdot C^{+}\right) \\
& =4 \pi \sum_{\jmath}\left([\omega] \cdot E_{\jmath}\right)\langle t\rangle_{\jmath}+4 \pi\left(\mathbf{a}[\omega] \cdot C^{-}+\mathbf{b}[\omega] \cdot C^{+}\right) \\
& =4 \pi \sum_{\imath}\langle t\rangle_{\imath} \mathcal{A}_{\imath}=2 \sum_{\imath}\langle x\rangle_{\imath} \mathcal{A}_{\imath}
\end{aligned}
$$

where $\mathcal{A}_{\imath}=[\omega] \cdot C_{\imath}$ is once again the area of $C_{\imath}$. Since the holomorphy potential of the holomorphic vector field $\Xi$ is $x=2 \pi t$, we therefore have

$$
\begin{aligned}
-\mathfrak{F}(\Xi,[\omega]) & =\int_{M} s(x-\bar{x}) d \mu \\
& =2 \pi \int_{M} s t d \mu-\bar{x} \int_{M} s d \mu \\
& =\left(4 \pi \sum_{\imath}\langle x\rangle_{\imath} \mathcal{A}_{\imath}\right)-\bar{x}\left(4 \pi c_{1} \cdot[\omega]\right)
\end{aligned}
$$

where $\bar{x}$ again denotes the average value of $x$ on $M$.

Next, notice that $\cup_{2} C_{\imath}$ is the zero locus of the holomorphic section $\Xi_{1} \wedge \Xi_{2}$ of the anti-conical line-bundle $K^{-1}=\wedge^{2} T^{1,0}$, and that, since the imaginary parts of $\Xi_{1}$ and $\Xi_{2}$ are Killing fields, this section is transverse to the zero section away from the intersection points $C_{\imath} \cap C_{\jmath}$. It follows that the homology 
class of $\cup_{\imath} C_{\imath}$ is Poincaré dual to $c_{1}(M, J)=c_{1}\left(K^{-1}\right)$. Hence

$$
c_{1} \cdot[\omega]=\sum_{\imath} C_{\imath} \cdot[\omega]=\sum_{\imath} \mathcal{A}_{\imath}
$$

so that (5.3) simplifies to become

$$
\mathfrak{F}(\Xi,[\omega])=-4 \pi \sum_{\imath}\left(\langle x\rangle_{\imath}-\bar{x}\right) \mathcal{A}_{\imath}
$$

and (5.2) therefore follows by setting $\Xi=\Xi_{k}$ and $x=x_{k}$.

With this preparation, we can now calculate $\mathcal{A}(\Omega)$ for any toric surface.

Proof of TheOrem 5.1. Relative to the basis given by the normalized holomorphy potentials $\left\{x_{k}-\bar{x}_{k} \mid k=1,2\right\}$, Proposition 5.2 tells us that the restriction of the Futaki invariant to $\mathfrak{t}$ is given by

$$
\overrightarrow{\mathfrak{F}}=\left(\mathfrak{F}_{1}, \mathfrak{F}_{2}\right)=-4 \pi|\partial P| \overrightarrow{\mathfrak{D}} .
$$

Since the $L^{2}$ inner product $\mathbb{B}_{\mathbf{T}}$ on $\mathfrak{t}$ is given in this basis by the moment-ofinertia matrix

$$
\Pi=\left[\int_{P}\left(x_{j}-\bar{x}_{j}\right)\left(x_{k}-\bar{x}_{k}\right) d a\right]=\left[\int_{M}\left(x_{j}-\bar{x}_{j}\right)\left(x_{k}-\bar{x}_{k}\right) d \mu\right],
$$

the dual inner product $\mathbb{B}_{\mathbf{T}}^{-1}$ on $\mathfrak{t}^{*}$ is represented by the inverse matrix $\Pi^{-1}$, and Proposition 3.4 therefore tells us that

$$
\|\mathfrak{F}\|^{2}=\overrightarrow{\mathfrak{F}} \cdot \Pi^{-1} \overrightarrow{\mathfrak{F}}=16 \pi^{2}|\partial P|^{2} \overrightarrow{\mathfrak{D}} \cdot \Pi^{-1} \overrightarrow{\mathfrak{D}}
$$

Since the first Chern class is Poincaré dual to the homology class of $\cup C_{\imath}$,

$$
c_{1} \cdot[\omega]=\sum_{\imath} C_{\imath} \cdot[\omega]=\sum_{\imath} \mathcal{A}_{\imath}=|\partial P|,
$$

while $M$ has volume $|P|=[\omega]^{2} / 2$. Thus

$$
\mathcal{A}(\Omega)=\frac{\left(c_{1} \cdot[\omega]\right)^{2}}{[\omega]^{2}}+\frac{1}{32 \pi^{2}}\|\mathfrak{F}\|^{2}=\frac{|\partial P|^{2}}{2}\left(\frac{1}{|P|}+\overrightarrow{\mathfrak{D}} \cdot \Pi^{-1} \overrightarrow{\mathfrak{D}}\right)
$$

exactly as claimed.

By (4.5), Theorem A is now an immediately immediate corollary.

\section{The Abreu Formalism}

The proof of Theorem A given in $\S 5$ was based on the author's formula [34] for the scalar curvature of Kähler surfaces with isometric $S^{1}$ actions. This section will present a different proof, which is based on Abreu's beautiful formula $[\mathbf{1}]$ for the scalar curvature of a toric manifold, and makes crucial use of an integration-by-parts trick due to Donaldson [21]. While this second proof is certainly more elegant and natural, there are unfortunately many numerical factors involved in this formalism that are typically misreported in the literature, and we will need to correct these imprecisions in order to obtain our result. This will be well worth the effort, 
however, insofar as this second proof works equally well in all complex dimensions. The reader should note, however, that the higher-dimensional version of Theorem A is of much less differential-geometric interest than the corresponding statement in complex dimension 2 ; it is only in real dimension 4 that the Calabi energy is intimately tied to the Weyl functional and conformally Einstein metrics.

We thus begin by considering a toric manifold $\left(M^{2 m}, J, \mathbf{T}\right)$ of complex dimension $m$, equipped with a Kähler metric $g$ which is invariant under the action of the $m$-torus $\mathbf{T} \cong T^{m}$. Choosing an isomorphism $\mathbf{T} \cong \mathbb{R}^{m} / \mathbb{Z}^{m}$, we then let $\left(\xi_{1}, \ldots, \xi_{m}\right)$ be the $m$ unit-period vector fields generating $\mathbf{T}$ associated with this choice, and let $\left(\Xi_{1}, \ldots, \Xi_{m}\right)$ be the holomorphic vector fields defined by $\Xi_{j}=\xi_{j}^{1,0}$. Let $\left(x_{1}, \ldots, x_{m}\right)$ be Hamiltonians for $\left(\xi_{1}, \ldots, \xi_{m}\right)$, and note that these are consequently also holomorphy potentials for $\left(\Xi_{1}, \ldots, \Xi_{m}\right)$. The function $\vec{x}: M \rightarrow \mathbb{R}^{m}$ given by $\left(x_{1}, \ldots, x_{m}\right)$ is then a moment map for this $T^{m}$-action, and its image $\vec{x}(M)$ is called the associated moment polytope. Once again, the moment polytope has the Delzant property: a neighborhood of any vertex $\in P$ can be transformed into a neighborhood of $\overrightarrow{0} \in[0, \infty)^{m}$ by an element of $\mathbf{S L}(m, \mathbb{Z}) \ltimes \mathbb{R}^{m}$. The $2 m$ dimensional volume measure on $M$ now pushes forward, by integration on the fibers, to the standard $m$-dimensional Euclidean measure on $\mathbb{R}^{m}$, which we will again denote by $d a$ to emphasize our special interest in the case of $m=2$. The boundary $\partial P$ is the image of a union of toric complex hypersurfaces in $M$, and the push-forward of $(2 m-2)$-dimensional Riemannian measure induces an $(m-1)$-dimensional measure $d \lambda$ on $\partial P$ which, on each face, is $\mathbf{S L}(n, \mathbb{Z})$-equivalent to the standard $(m-1)$-dimensional Euclidean measure on the hyper-plane $x_{1}=0$.

For consistency with $[\mathbf{1}, \mathbf{2 1}]$, it will be convenient to also consider the vector fields $\eta_{j}=\xi_{j} / 2 \pi$ of period $2 \pi$, and their Hamiltonians $t^{j}=x_{j} / 2 \pi$; the corresponding moment map is then $\vec{t}=\left(t^{1}, \ldots, t^{m}\right)$, and its image $\tilde{P}=\vec{t}(M)$ can then be transformed into $P$ by dilating by a factor of $2 \pi$. Following Donaldson, we will use $d \mu$ to denote $m$-dimensional Euclidean measure on $\tilde{P}$, and $d \sigma$ to denote the $(m-1)$-dimensional measure on $\partial \tilde{P}$ which, on each face, is $\mathbf{S L}(n, \mathbb{Z})$-equivalent to $(m-1)$-dimensional Euclidean measure on the hyperplane $t^{1}=0$. Identifying $\tilde{P}$ with $P$ via the obvious homothety, we thus have $d a=(2 \pi)^{m} d \mu$ and $d \lambda=(2 \pi)^{m-1} d \sigma$.

On the open dense set $\vec{t}^{-1}(\operatorname{Int} \tilde{P}) \subset M$, Abreu observed that our $T^{m_{-}}$ invariant Kähler metric can be expressed as

$$
g=V_{, j k} d t^{j} \otimes d t^{k}+V^{, j k} d \vartheta_{j} \otimes d \vartheta_{k}
$$

where $V: \tilde{P} \rightarrow \mathbb{R}$ is a convex potential function, $\left[V_{, j k}\right]$ is the Hessian matrix of $V,\left[V^{, j k}\right]$ is its inverse matrix, and the $\vartheta_{j}$ are standard angle coordinates on $T^{m}=S^{1} \times \cdots \times S^{1}$. The potential $V$ is Legendre dual to a Kähler potential for $g$; it is continuous on $\tilde{P}$ and smooth in its interior. Moreover, it satisfies the so-called Guillemin-Abreu boundary condition: near a face 
given by $L=0$, where the affine linear function $L: \mathbb{R}^{m} \rightarrow \mathbb{R}$ is non-negative on $\tilde{P}$ and where $d L$ is an indivisible element of the integer lattice $\left(\mathbb{Z}^{m}\right)^{*}, V$ differs from $\frac{1}{2} L \log L$ by a smooth function. (Note that the factor of $1 / 2$ is missing from [21, p. 303], and will lead to a compensating correction below.) The scalar curvature $s$ of $g$ is then expressible in terms of $V$ via Abreu's beautiful formula $[\mathbf{1}, \mathbf{2 1}]$

$$
s=-\left(V^{, j k}\right)_{, j k}:=-\sum_{j, k=1}^{m} \frac{\partial^{2} V^{, j k}}{\partial t_{j} \partial t_{k}},
$$

where we have followed Donaldson's conventions in order to give $s$ its standard Riemannian value.

In this setting, Donaldson [21, Lemma 3.3.5] derives the integration-byparts formula

$$
\int_{\tilde{P}} V^{, j k} f_{, j k} d \mu=\int_{\tilde{P}}\left(V^{, j k}\right)_{, j k} f d \mu+2 \int_{\partial \tilde{P}} f d \sigma
$$

for any convex function $f$. Note, however, that the factor of 2 in front of the boundary term does not actually appear in [21], but is needed to compensate for the factor of $1 / 2$ in the corrected Abreu-Guillemin boundary conditions. We also give the boundary term a different sign, because we are treating $d \sigma$ as a measure rather than as an exterior differential form.

Example Let $(M, g)$ be the unit 2-sphere, with sectional curvature $K=1$, and hence with scalar curvature $s=2 K=2$. Equip $(M, g)$ with the $S^{1}$ action given by period- $2 \pi$ rotation around the the $z$-axis, with Hamiltonian $t=z$ and moment polytope $\tilde{P}=[-1,1]$. In cylindrical coordinates, our metric becomes

$$
g=\frac{d t^{2}}{1-t^{2}}+\left(1-t^{2}\right) d \vartheta^{2}
$$

so that the potential $V$ must satisfy $V_{, 11}=1 /\left(1-t^{2}\right)$ and $V^{, 11}=1-t^{2}$. A suitable choice of $V$ is therefore

$$
V=\frac{1}{2}(1+t) \log (1+t)+\frac{1}{2}(1-t) \log (1-t)
$$

and we note that this satisfies the Guillemin-Abreu boundary conditions discussed above. The Abreu formula (6.1) now correctly calculates the scalar curvature

$$
s=-\left(V^{, 11}\right)_{, 11}=-\frac{d^{2}}{d t^{2}}\left(1-t^{2}\right)=2
$$

of $g$. Also notice that integration by parts gives

$$
\int_{-1}^{1}\left(1-t^{2}\right) f^{\prime \prime} d t=\int_{-1}^{1}\left(1-t^{2}\right)^{\prime \prime} f d t+2[f(-1)+f(1)]
$$

as predicted by (6.2). 
Example Let $\left(M^{2 m}, g\right)$ be the Riemannian product $S^{2} \times \cdots \times S^{2}$ of $m$ copies of the unit 2 -sphere, with equipped with the product $T^{m}$-action. The moment polytope is now the $m$-cube $\tilde{P}=[-1,1]^{m}$, and the metric is again represented by a symplectic potential

$$
V=\frac{1}{2} \sum_{j}\left[\left(1+t^{j}\right) \log \left(1+t^{j}\right)+\left(1-t^{j}\right) \log \left(1-t^{j}\right)\right]
$$

which satisfies our corrected Guillemin-Abreu boundary conditions. The Abreu formula (6.1) now predicts that the scalar curvature of $g$ is

$$
s=-\left(V^{, i j}\right)_{, i j}=-\sum_{j} \frac{\partial^{2}}{\partial\left(t^{j}\right)^{2}}\left(1-t_{j}^{2}\right)=2 m,
$$

in agreement with the additivity of the scalar curvature under Riemannian products. Integrating the $j^{\text {th }}$ term by parts twice in the $j^{\text {th }}$ variable, we have

$$
\int_{\tilde{P}}\left[\sum_{j}\left[1-\left(t^{j}\right)^{2}\right] \frac{\partial^{2} f}{\partial\left(t^{j}\right)^{2}}\right] d \mu=\int_{\tilde{P}}\left[\sum_{j} \frac{\partial^{2}\left[1-\left(t^{j}\right)^{2}\right]}{\partial\left(t^{j}\right)^{2}}\right] f d \mu+2 \int_{\partial \tilde{P}} f d \sigma,
$$

for any smooth $f$, thereby double-checking (6.2) in complex dimension $m$.

By linearity, (6.2) also holds [21, Corollary 3.3.10] if $f$ is any difference of convex functions. In particular, (6.2) applies to any affine linear function $f$ on $\mathbb{R}^{m}$; and since any such $f$ satisfies $f_{, j k}=0,(6.1)$ and (6.2) tell us that

$$
0=\int_{\tilde{P}}(-s) f d \mu+2 \int_{\partial \tilde{P}} f d \sigma
$$

for any affine-linear function. Applying the dilation that relates $\tilde{P}$ and $P$, we therefore obtain

$$
\int_{P} s f d a=4 \pi \int_{\partial P} f d \lambda
$$

for any affine-linear $f$. In particular, if we take $f=x_{k}-\bar{x}_{k}$, we obtain

$$
\int_{P} x_{k}(s-\bar{s}) d a=\int_{P}\left(x_{k}-\bar{x}_{k}\right) s d a=4 \pi \int_{\partial P}\left(x_{k}-\bar{x}_{k}\right) d \lambda
$$

which in turn implies that

$$
\int_{M} x_{k}(s-\bar{s}) d \mu=4 \pi \int_{\partial P}\left(x_{k}-\bar{x}_{k}\right) d \lambda
$$

because $d a$ is the push-forward of the volume measure of $(M, g)$. However, $x_{k}$ is a holomorphy potential for the holomorphic vector field $\Xi_{k}$, so $(2.1)$ tells us that the component

$$
\mathfrak{F}_{k}:=\mathfrak{F}\left(\Xi_{k}, \Omega\right)
$$


of the Futaki invariant is given by

$$
\mathfrak{F}_{k}=-4 \pi \int_{\partial P}\left(x_{k}-\bar{x}_{k}\right) d \lambda .
$$

On the other hand,

$$
\frac{1}{|\partial P|} \int_{\partial P}\left(x_{k}-\bar{x}_{k}\right) d \lambda=\left\langle x_{k}-\bar{x}_{k}\right\rangle=\left\langle x_{k}\right\rangle-\bar{x}_{k}=\mathfrak{D}_{k}
$$

where $|\partial P|$ denotes the $\lambda$-measure of the boundary, \langle\rangle is the average with respect to $d \lambda$, and where $\mathfrak{D}_{k}$ is the $k^{\text {th }}$ component of the vector $\mathfrak{D}$ which points from the barycenter of $P$ to the barycenter of $\partial P$. Thus the Futaki invariant $\mathfrak{F}(\Omega)=\overrightarrow{\mathfrak{F}}=\left(\mathfrak{F}_{1}, \ldots, \mathfrak{F}_{m}\right)$ is given by

$$
\overrightarrow{\mathfrak{F}}=-4 \pi|\partial P| \overrightarrow{\mathfrak{D}}
$$

and we have thus reproved Proposition 5.2 in arbitrary complex dimension $m$.

Now notice that, by taking normalized Hamiltonians, the Lie algebra $\mathfrak{t}$ of our maximal torus $\mathbf{T}$ is naturally identified with those affine-linear functions $\mathbb{R}^{m} \rightarrow \mathbb{R}$ which send the barycenter $\overline{\vec{x}}$ of our moment polytope to 0 . From this view-point, it is now apparent that $\mathfrak{F}(\Omega)=-4 \pi|\partial P| \overrightarrow{\mathfrak{D}}$ actually belongs to $\mathfrak{t}^{*}$, as it should. In these same terms, though, the "moment-of inertia" matrix $\Pi$ defined by

$$
\Pi_{j k}=\int_{P}\left(x_{j}-\bar{x}_{j}\right)\left(x_{k}-\bar{x}_{k}\right) d a
$$

represents the $L^{2}$ inner product

$$
\mathbb{B}_{\mathbf{T}}: \mathfrak{t} \times \mathfrak{t} \rightarrow \mathbb{R},
$$

while its inverse matrix $\Pi^{-1}$ represents the dual inner product

$$
\mathbb{B}_{\mathbf{T}}^{-1}: \mathfrak{t}^{*} \times \mathfrak{t}^{*} \rightarrow \mathbb{R}
$$

By Corollary 2.3 and (6.4), we thus have

$$
\|\mathfrak{F}(\Omega)\|^{2}=\mathbb{B}_{\mathbf{T}}^{-1}(\mathfrak{F}(\Omega), \mathfrak{F}(\Omega))=16 \pi^{2}|\partial P|^{2} \overrightarrow{\mathfrak{D}} \cdot \Pi^{-1} \overrightarrow{\mathfrak{D}} .
$$

Chen's inequality (4.1) therefore tells us ${ }^{2}$ that any Kähler metric on a toric manifold satisfies

$$
\int_{M}(s-\bar{s})^{2} d \mu \geq 16 \pi^{2}|\partial P|^{2} \overrightarrow{\mathfrak{D}} \cdot \Pi^{-1} \overrightarrow{\mathfrak{D}}
$$

where the moment polytope $P$ is determined solely by the toric manifold $M$ and the Kähler class $\Omega$; moreover, equality holds iff $g$ is extremal.

On the other hand, setting $f=1$ in (6.3) yields

$$
\int_{P} s d a=4 \pi \int_{\partial P} d \lambda
$$

\footnotetext{
${ }^{2}$ Here it is worth reiterating that, while the inequality (4.1) is essentially elementary when $g$ is $\mathbf{T}$-invariant, it is a deep and remarkable result that this same inequality in fact holds for completely arbitrary Kähler metrics.
} 
so that

$$
\int_{M} s d \mu=4 \pi|\partial P|
$$

a fact which the reader may enjoy comparing with (4.3). Since $(M, g)$ has volume $|P|$, we therefore see that

$$
\int_{M} \bar{s}^{2} d \mu=\frac{\left(\int_{M} s d \mu\right)^{2}}{\int_{M} d \mu}=16 \pi^{2} \frac{|\partial P|^{2}}{|P|}
$$

and the Pythagorean theorem (4.2) therefore implies the following result:

TheOREM B. Let $\left(M^{2 m}, J, \Omega, \mathbf{T}\right)$ be a toric complex $m$-manifold with fixed Kähler class, and let $P \subset \mathbb{R}^{m}$ be the associated moment polytope. Then the scalar curvature $s$ of any Kähler metric $g$ with Kähler form $\omega \in \Omega$ satisfies

$$
\frac{1}{16 \pi^{2}} \int_{M} s^{2} d \mu_{g} \geq|\partial P|^{2}\left(\frac{1}{|P|}+\overrightarrow{\mathfrak{D}} \cdot \Pi^{-1} \overrightarrow{\mathfrak{D}}\right),
$$

with equality iff $g$ is an extremal Kähler metric. Here $|P|$ denotes the $m$ volume of the interior of $P,|\partial P|$ is the $\lambda$-volume of its boundary, the moment-of-inertia matrix $\Pi$ of $P$ is defined by (6.5), and $\overrightarrow{\mathfrak{D}}$ is the vector joining the barycenter $P$ to the barycenter of $\partial P$.

Specializing to the case of $m=2$ gives a second proof of Theorem A.

Notice that the sharp lower bound (6.6) is in fact independent of dimension. However, this feature of the result actually depends on our conventions regarding the moment polytope and the generators of the action. For example, if we had instead chosen the periodicity of our generators to be $2 \pi$ instead of 1 , we would have been led to instead use the polytope $\tilde{P}$, and we would have then been forced to introduce an inconvenient scaling factor, since

$$
\frac{|\partial P|^{2}}{|P|}=(2 \pi)^{m-2} \frac{|\partial \tilde{P}|^{2}}{|\tilde{P}|}
$$

But it is also worth noticing that this awkward scaling factor magically disappears when $m=2$. This reflects the fact that the Calabi energy is invariant under rescaling in real dimension four, and that rescaling a Kähler class exactly results in a rescaling of the associated moment polytope.

In particular, for the purpose of calculating the virtual action $\mathcal{A}$ for toric surfaces, we would have obtained exactly the same formula if we had used the rescaled polygon $\tilde{P}$ instead of the polygon $P$ emphasized by this article. Nonetheless, the use of $P$ has other practical advantages, even when $m=2$. For example, the $\lambda$-length of sides of $P$ directly represents the areas of holomorphic curves in $M$, unmediated by factors of $2 \pi$. In practice, this avoids repeatedly having to cancel powers of $2 \pi$ when calculating $\mathcal{A}(\Omega)$ in explicit examples. This will now become apparent, as we next illustrate Theorem A by applying it to specific toric surfaces. 


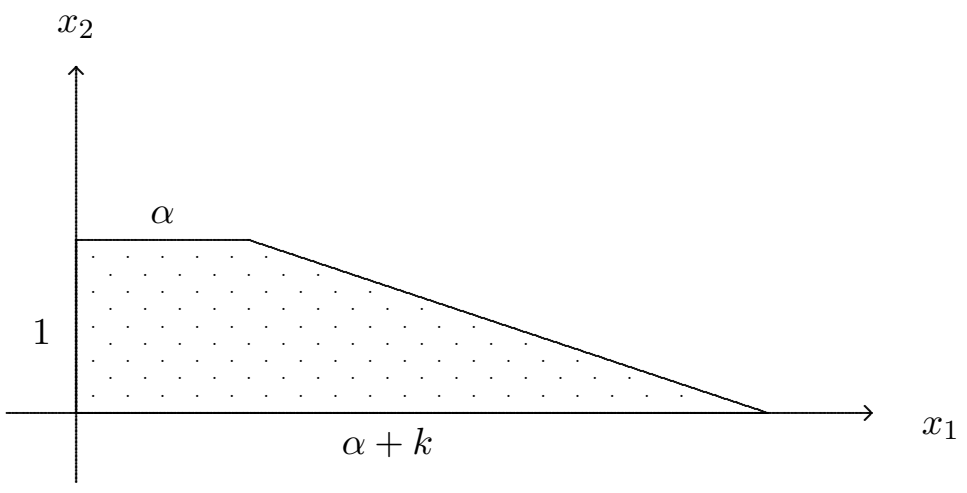

FiguRE 1

\section{Hirzebruch Surfaces}

As a simple illustration of Theorem 5.1, we now compute $\mathcal{A}(\Omega)$ for the Hirzebruch surfaces. Recall $[\mathbf{7}, \mathbf{2 6}]$ that, for any non-negative integer $k$, the $k^{\text {th }}$ Hirzebruch surface $\mathbb{F}_{k}$ is defined to be the $\mathbb{C P}_{1}$-bundle $\mathbb{P}(\mathcal{O}(k) \oplus \mathcal{O})$ over $\mathbb{C P}_{1}$; that is, it is the complex surface obtained from line bundle $\mathcal{O}(k) \rightarrow \mathbb{C P}_{1}$ of Chern class $k$ by adding a section at infinity. Calabi $[\mathbf{1 1}]$ explicitly constructed an extremal every Kähler metric in every Kähler class on each $\mathbb{F}_{k}$; his direct assault on the problem proved feasible because the maximal compact subgroup $\mathbf{U}(2) / \mathbb{Z}_{k}$ of the automorphism group has orbits of real codimension 1, thereby reducing the relevant equation for the Kähler potential to an ODE. Because their automorphism groups all contain finite quotients of $\mathbf{U}(2)$, the Hirzebruch surfaces all admit actions of the 2-torus $T^{2}$, and so are toric surfaces. Normalizing the fibers of $\mathbb{F}_{k} \rightarrow \mathbb{C P}_{1}$ to have area 1 , the associated moment polygon becomes the trapezoid shown in Figure 1 and since $\mathcal{A}(\Omega)$ is unchanged by multiplying $\Omega$ by a positive constant, we may impose this normalization without loss of generality.

We will now apply Theorem 5.1 to calculate the Calabi energy of Calabi's extremal Kähler metrics; since Hwang and Simanca [30] have previously computed this quantity by other means, this exercise will, among other things, provide us with another useful double-check of equation (5.1). The area and $\lambda$-perimeter of the polygon are easily seen to be

$$
|P|=\alpha+\frac{k}{2},|\partial P|=2+2 \alpha+k
$$

and it is not difficult to calculate the barycenter of the interior

$$
\overline{\vec{x}}=\frac{\left(3 \alpha^{2}+3 k \alpha+k^{2}, 3 \alpha+k\right)}{6|P|}
$$


or boundary

$$
\langle\vec{x}\rangle=\frac{\left(\alpha^{2}+\alpha(k+1)+\frac{1}{2} k(k+1), \alpha+1\right)}{|\partial P|}
$$

by hand. The vector

$$
\overrightarrow{\mathfrak{D}}=\frac{k(2 \alpha+k-1)}{12|\partial P||P|}(k,-2)
$$

thus joins these two barycenter, and without too much work one can also check that the "moment-of inertia" matrix of $P$ is given by

$$
\Pi=\frac{1}{72|P|}\left[\begin{array}{cc}
6 \alpha^{4}+12 \alpha^{3} k+12 \alpha^{2} k^{2}+6 \alpha k^{3}+k^{4} & -\frac{k}{2}\left(6 \alpha^{2}+6 \alpha k+k^{2}\right) \\
-\frac{k}{2}\left(6 \alpha^{2}+6 \alpha k+k^{2}\right) & 6 \alpha^{2}+6 \alpha k+k^{2}
\end{array}\right]
$$

The Futaki contribution to $\mathcal{A}$ is therefore encoded by the expression

$$
\overrightarrow{\mathfrak{D}} \cdot \Pi^{-1} \overrightarrow{\mathfrak{D}}=\frac{2 k^{2}(2 \alpha+k-1)^{2}}{|P \| \partial P|^{2}\left(6 \alpha^{2}+6 \alpha k+k^{2}\right)}
$$

and the virtual action is thus given by

$$
\mathcal{A}(\Omega)=\frac{2 \alpha^{3}+(4+3 k) \alpha^{2}+2(1+k)^{2} \alpha+k\left(k^{2}+2\right) / 2}{\alpha^{2}+\alpha k+k^{2} / 6} .
$$

After multiplication by an overall constant and the change of variables $k=n$, $\alpha=(a-n) / 2$, this agrees with with the expression Hwang and Simanca [30, equation (3.2)] obtained for their "potential energy" via a different method.

For $k>0$, the function $\mathcal{A}(\alpha)$ on the right-hand side of (7.1) extends smoothly across $\alpha=0$, and satisfies

$$
\left.\frac{d \mathcal{A}}{d \alpha}\right|_{\alpha=0}=-6 \frac{(k-2)^{2}}{k}
$$

so $\mathcal{A}(\alpha)$ is a decreasing function for small $\alpha$ if $k \neq 2$. On the other hand, $\mathcal{A}(\alpha) \sim 2 \alpha$ for $\alpha \gg 0$, so $\mathcal{A}$ is increasing for large $\alpha$. It follows that $\mathcal{A}(\alpha)$ has a minimum somewhere on $\mathbb{R}^{+}$for any $k \neq 2$. Since Calabi's construction [11] moreover shows that each Kähler class on a Hirzebruch surface is represented by an extremal Kähler metric, Proposition 4.1 tells us that, for $k \neq 2$, the Calabi metric $g_{k}$ corresponding to the minimizing value of $\alpha$ is necessarily Bach-flat.

On the other hand, since

$$
\mathcal{A}(\Omega)-\frac{3}{4} k=\frac{48 \alpha^{3}+(54 k+96) \alpha^{2}+\left(30 k^{2}+96 k+48\right) \alpha+9 k^{3}+24 k}{4\left(6 \alpha^{2}+6 k \alpha+k^{2}\right)}
$$

is positive for all $\alpha>0$, it follows that

$$
\min _{\Omega} \mathcal{A}(\Omega)>\frac{3}{4} k
$$

and we conclude that the corresponding Bach-flat Kähler metric $g_{k}$ has

$$
\mathcal{W}\left(g_{k}\right)>2 \pi^{2} k
$$


Since the Hirzebruch surface $\mathbb{F}_{k}$ is diffeomorphic to $S^{2} \times S^{2}$ when $k$ is even, and is diffeomorphic to $\mathbb{C P}_{2} \# \overline{\mathbb{C P}}_{2}$ when $k$ is odd, the metrics $g_{k}$, first discovered by Hwang and Simanca [30], immediately give us the following:

Proposition 7.1. The smooth 4-manifolds $S^{2} \times S^{2}$ and $\mathbb{C P}_{2} \# \overline{\mathbb{C P}}_{2}$ both admit sequences of Bach-flat conformal classes $\left[g_{k_{j}}\right]$ with $\mathcal{W}\left(\left[g_{k_{j}}\right]\right) \rightarrow+\infty$. Consequently, the moduli space of Bach-flat conformal metrics on either of these manifolds has infinitely many connected components.

The metric $g_{1}$ on $\mathbb{F}_{1}$ has scalar curvature $s>0$ everywhere, and its conformal rescaling $s^{-2} g_{1}$ was shown by Derdziński $[\mathbf{1 9}]$ to coincide with the Einstein metric on $\mathbb{C P}_{2} \# \overline{\mathbb{C P}}_{2}$ discovered by Page [48]. For $k \geq 3$, the scalar curvature $s$ of $g_{k}$ instead vanishes along a hypersurface, which becomes the conformal infinity for the Einstein metric $s^{-2} g_{k}$; thus $\mathbb{F}_{k}$ is obtained from two Poincaré-Einstein manifolds, glued along their conformal infinity. These two Einstein metrics are in fact isometric, in an orientation-reversing manner. Because of their $\mathbf{U}(2)$ symmetry, these Einstein metrics belong to the family first discovered by Bérard-Bergery [8], and later rediscovered by physicists, who call them AdS-Taub-bolt metrics [29].

\section{The Two-Point Blow-Up of $\mathbb{C P}_{2}$}

As a final illustration of Theorem 5.1, we now compute the virtual action for Kähler classes on the blow-up of $\mathbb{C P}_{2}$ at two distinct points. The present author has done this elsewhere by a more complicated method, and the details of the answer played an important role in showing $[\mathbf{1 5}, \mathbf{3 7}]$ that this manifold admits an Einstein metric, obtained by conformally rescaling a Bach-flat Kähler metric. Thus, repeating the computation by means of equation (5.1) provides yet another double-check of Theorem A.

Blowing up $\mathbb{C P}_{2}$ in two distinct points results in exactly the same complex surface as blowing $\mathbb{C P}_{1} \times \mathbb{C P}_{1}$ in a single point $[\mathbf{7 , 2 6}]$. The latter

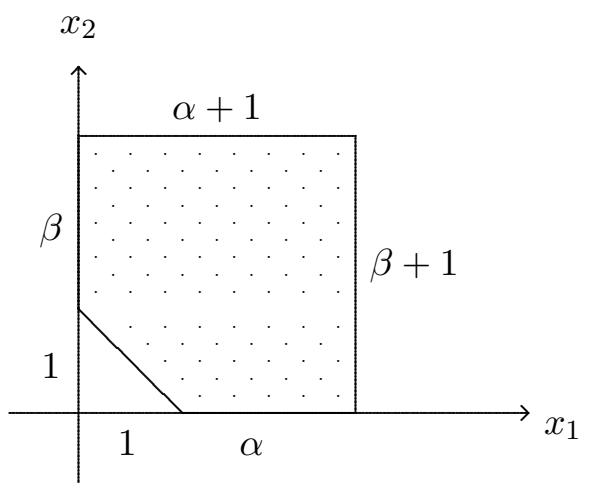

FiguRE 2 
picture is actually useful in choosing a pair of generators for the torus action which makes the needed computations as simple as possible. The resulting moment polygon $P$ then takes the form shown in Figure 2 after rescaling to give the blow-up divisor area 1 . It is then easy to see that the area of the polygon and the $\lambda$-length of its boundary are given by

$$
|P|=\frac{1}{2}+\alpha+\beta+\alpha \beta, \quad|\partial P|=3+2 \alpha+2 \beta
$$

while the barycenter of the boundary

$$
\langle\vec{x}\rangle=\frac{((1+\alpha)(2+\alpha+\beta), \quad(1+\beta)(2+\alpha+\beta))}{|\partial P|}
$$

and of the interior

$$
\overline{\vec{x}}=\frac{\left(3(1+\alpha)^{2}(1+\beta)-1, \quad 3(1+\alpha)(1+\beta)^{2}-1\right)}{6|P|}
$$

are not difficult to compute by hand. The vector joining these two barycenters is thus given by

$$
\overrightarrow{\mathfrak{D}}=\frac{\left(-\alpha+2 \beta+3 \alpha \beta+3 \alpha^{2} \beta, \quad-\beta+2 \alpha+3 \alpha \beta+3 \alpha \beta^{2}\right)}{6|P||\partial P|}
$$

and the moment-of-inertia matrix

$\Pi=\frac{1}{24}\left[\begin{array}{cc}8(1+\alpha)^{3}(1+\beta)-2 & 6(1+\alpha)^{2}(1+\beta)^{2}-1 \\ 6(1+\alpha)^{2}(1+\beta)^{2}-1 & 8(1+\alpha)(1+\beta)^{3}-2\end{array}\right]-|P|\left[\begin{array}{cc}\bar{x}_{1}^{2} & \bar{x}_{1} \bar{x}_{2} \\ \bar{x}_{1} \bar{x}_{2} & \bar{x}_{1}^{2}\end{array}\right]$ are also easily obtained without the use of a computer. According to (5.1), $\mathcal{A}(\Omega)$ is therefore given by

$$
\begin{aligned}
& 3\left[3+28 \beta+96 \beta^{2}+168 \beta^{3}+164 \beta^{4}+80 \beta^{5}+16 \beta^{6}+16 \alpha^{6}(1+\beta)^{4}+16 \alpha^{5}(5+24 \beta+\right. \\
& \left.43 \beta^{2}+37 \beta^{3}+15 \beta^{4}+2 \beta^{5}\right)+4 \alpha^{4}\left(41+228 \beta+478 \beta^{2}+496 \beta^{3}+263 \beta^{4}+60 \beta^{5}+4 \beta^{6}\right)+ \\
& 8 \alpha^{3}\left(21+135 \beta+326 \beta^{2}+392 \beta^{3}+248 \beta^{4}+74 \beta^{5}+8 \beta^{6}\right)+4 \alpha\left(7+58 \beta+176 \beta^{2}+270 \beta^{3}+\right. \\
& \left.\left.228 \beta^{4}+96 \beta^{5}+16 \beta^{6}\right)+4 \alpha^{2}\left(24+176 \beta+479 \beta^{2}+652 \beta^{3}+478 \beta^{4}+172 \beta^{5}+24 \beta^{6}\right)\right] / \\
& {\left[1+10 \beta+36 \beta^{2}+64 \beta^{3}+60 \beta^{4}+24 \beta^{5}+24 \alpha^{5}(1+\beta)^{5}+12 \alpha^{4}(1+\beta)^{2}(5+20 \beta+\right.} \\
& \left.23 \beta^{2}+10 \beta^{3}\right)+16 \alpha^{3}\left(4+28 \beta+72 \beta^{2}+90 \beta^{3}+57 \beta^{4}+15 \beta^{5}\right)+12 \alpha^{2}\left(3+24 \beta+69 \beta^{2}+\right. \\
& \left.\left.96 \beta^{3}+68 \beta^{4}+20 \beta^{5}\right)+2 \alpha\left(5+45 \beta+144 \beta^{2}+224 \beta^{3}+180 \beta^{4}+60 \beta^{5}\right)\right]
\end{aligned}
$$

as is most easily checked at this point using Mathematica or a similar program. After the substitution $\gamma=\alpha$, this agrees exactly with the answer obtained in $[\mathbf{3 8}, \S 2]$, where this explicit formula plays a key role in classifying compact Einstein 4-manifolds for which the metric is Hermitian with respect to some complex structure.

When $\alpha=\beta$, the above expression simplifies to become

$$
\frac{9+96 \alpha+396 \alpha^{2}+840 \alpha^{3}+954 \alpha^{4}+528 \alpha^{5}+96 \alpha^{6}}{1+12 \alpha+54 \alpha^{2}+120 \alpha^{3}+138 \alpha^{4}+72 \alpha^{5}+12 \alpha^{6}}
$$


which, after dividing by 3 and making the substitution $\alpha=1 / y$, coincides with the expression [36] first used to show that $\mathcal{A}$ has a critical point, and later used again [15] to prove the existence of a conformally Einstein, Kähler metric on $\mathbb{C P}_{2} \# 2 \overline{\mathbb{C P}}_{2}$. For a second, conceptually simpler proof of this last fact, see $[\mathbf{3 7}]$.

\section{Appendix A. Restricting the Futaki Invariant}

In this appendix, we will prove Proposition 2.1. The key ingredient used in the proof is the following result of Nakagawa [45]:

Proposition A.1 (Nakagawa). Let $(M, J)$ be a projective algebraic complex manifold, let $\mathbf{H}$ be the identity component of its complex automorphism group, and suppose that the Jacobi homomorphism from $\mathbf{H}$ to the Albanese torus of $M$ is trivial. Let $L \rightarrow M$ be an ample line bundle for which the action of $\mathbf{H}$ on $M$ lifts to an action on $L \rightarrow M$, and let $\Omega$ be the Kähler class defined by $\Omega=c_{1}(L)$. Then the Futaki invariant $\mathfrak{F}(\Omega) \in \mathfrak{h}^{*}$ annihilates the Lie algebra $\mathfrak{r}_{\mathfrak{u}}$ of the unipotent radical of $\mathbf{H}$.

This generalizes a previous result of Mabuchi [42] concerning the case when $L$ is the anti-canonical line bundle. Both of these results are proved using Tian's localization formula [53] for the Futaki invariant of a Hodge metric.

We will now extend Proposition A.1 to irrational Kähler classes on certain complex manifolds. In order to do this, we will first need the following observation:

Lemma A.2. Let $(M, J)$ be a compact complex manifold with $b_{1}(M)=0$, and let $\mathbf{H}$ be the identity component of its complex automorphism group. If $L \rightarrow M$ is a positive line bundle, then the action of $\mathbf{H}$ on $M$ lifts to an action on $L^{k} \rightarrow M$ for some positive integer $k$.

Proof. By the Kodaira embedding theorem $[\mathbf{2 6}], L$ has a positive power $L^{\ell}$ for which there is a canonical holomorphic embedding $j: M \hookrightarrow \mathbb{P}(\mathbb{V})$ such that $j^{*} \mathcal{O}(-1)=L^{-\ell}$, where $\mathbb{V}:=\left[H^{0}\left(M, \mathcal{O}\left(L^{\ell}\right)\right)\right]^{*}$.

Now since $(M, J)$ is of Kähler type and $H^{1}(M, \mathbb{C})=0$, the Hodge decomposition tells us that $H^{0,1}(M)=H^{1}(M, \mathcal{O})=0$, and the long exact sequence

$$
\cdots \rightarrow H^{1}(M, \mathcal{O}) \rightarrow H^{1}\left(M, \mathcal{O}^{\times}\right) \rightarrow H^{2}(M, \mathbb{Z}) \rightarrow \cdots
$$

therefore implies that holomorphic line bundles on $M$ are classified by their first Chern classes. On the other hand, since $\mathbf{H}$ is connected, each automorphism $\Phi: M \rightarrow M, \Phi \in \mathbf{H}$, is homotopic to the identity; and since Chern classes are homotopy invariants, we deduce that that $c_{1}\left(\Phi^{*} L\right)=c_{1}(L)$ for all $\Phi \in \mathbf{H}$. Consequently, $\Phi^{*} L \cong L$ as a holomorphic line bundle for any $\Phi \in \mathbf{H}$. While the resulting isomorphism $\Phi^{*} L \cong L$ is not unique, any two such isomorphisms merely differ by an overall multiplicative constant, and the associated linear map $H^{0}\left(M, \mathcal{O}\left(L^{\ell}\right)\right) \rightarrow H^{0}\left(M, \mathcal{O}\left(L^{\ell}\right)\right)$ induced by 
$\Phi^{*}$ is therefore completely determined up to an overall scale factor. Thus, for every $\Phi \in \mathbf{H}$, there is a uniquely determined projective transformation $\mathbb{P}(\mathbb{V}) \rightarrow \mathbb{P}(\mathbb{V})$, where again $\mathbb{V}:=\left[H^{0}\left(M, \mathcal{O}\left(L^{\ell}\right)\right)\right]^{*}$. This gives us a faithful projective representation $\mathbf{H} \hookrightarrow \mathbf{P S L}(\mathbb{V})$ which acts on $M \subset \mathbb{P}(\mathbb{V})$ via the original action of $\mathbf{H}$.

Now consider the group $\mathbf{S L}(\mathbb{V})$ of unit-determinant linear endomorphisms of $\mathbb{V}$, and observe that there is a short exact sequence

$$
0 \rightarrow \mathbb{Z}_{n} \rightarrow \mathbf{S L}(\mathbb{V}) \rightarrow \mathbf{P S L}(\mathbb{V}) \rightarrow 1
$$

where $n=\operatorname{dim} \mathbb{V}$; that is, every projective transformation of $\mathbb{P}(\mathbb{V})$ arises from $n$ different linear unit-determinant linear endomorphisms of $\mathbb{V}$, differing from each other merely by multiplication by an $n^{\text {th }}$ root of unity. If $\widetilde{\mathbf{H}}<\mathbf{S L}(\mathbb{V})$ is the inverse image of $\mathbf{H}<\mathbf{P S L}(\mathbb{V})$, then $\widetilde{\mathbf{H}}$ acts on $\mathbb{V}$, and so also acts on the tautological line bundle $\mathcal{O}(-1)$ over $\mathbb{P}(\mathbb{V})$. Restricting $\mathcal{O}(-1)$ to $M$ then gives us an action of $\widetilde{\mathbf{H}}$ on $L^{-\ell}$ which lifts the action of $\mathbf{H}$ on $M$, in such a manner that any two lifts of a given element only differ by multiplication of an $n^{\text {th }}$ root of unity. The induced action of $\widetilde{\mathbf{H}}$ on $L^{-n \ell}$ therefore descends to an action of $\mathbf{H}$, and passing to the dual line bundle $L^{n \ell}$ thus shows that the action of $\mathbf{H}$ on $M$ can be lifted to an action on $L^{k} \rightarrow M$ for $k=n \ell$.

Proposition A.3. Let $(M, J)$ be a compact complex manifold of Kähler type, and suppose that $M$ does not carry any non-trivial holomorphic 1-or 2 -forms. Then, for any Kähler class $\Omega$ on $M$, the Futaki invariant $\mathfrak{F}(\Omega) \in \mathfrak{h}^{*}$ annihilates the unipotent radical $\mathfrak{r}_{\mathfrak{u}} \subset \mathfrak{h}$.

Proof. By hypothesis, $H^{1,0}(M)=H^{2,0}(M)=0$. The Hodge decomposition therefore tells us that $b_{1}(M)=0$ and that $H^{1,1}(M, \mathbb{R})=H^{2}(M, \mathbb{R})$. Consequently, the Kähler cone $\mathcal{K} \subset H^{1,1}(M, \mathbb{R})$ is open in $H^{2}(M, \mathbb{R})$. Since $H^{2}(M, \mathbb{Q})$ is dense in $H^{2}(M, \mathbb{R})$, it follows that $H^{2}(M, \mathbb{Q}) \cap \mathcal{K}$ is dense in $\mathcal{K}$. In particular, $H^{2}(M, \mathbb{Q}) \cap \mathcal{K}$ is non-empty, and so, clearing denominators, we conclude that the Kähler cone $\mathcal{K}$ must meet the the integer lattice $H^{2}(M, \mathbb{Z})$ /torsion $\subset H^{2}(M, \mathbb{R})$. This argument, due to Kodaira [33], shows that $(M, J)$ carries Kähler metrics of Hodge type, and is therefore projective algebraic.

Pursuing this idea in the opposite direction, let $\Psi$ now be an integral Kähler class, so that $\Psi=c_{1}(L)$ for some positive line bundle $L \rightarrow M$. By Lemma A.2, the action of $\mathbf{H}$ on $M$ then lifts to some positive power $L^{k}$ of $L$. Since our hypotheses also imply that the Albanese torus is trivial, Proposition A.1 therefore implies that $\mathfrak{F}(k \Psi) \in \mathfrak{h}^{*}$ annihilates $\mathfrak{r}_{\mathfrak{u}}$. However, our expression (2.1) for the Futaki invariant implies that

$$
\mathfrak{F}(\Xi, \lambda \Omega)=\lambda^{m} \mathfrak{F}(\Xi, \Omega)
$$

for any $\lambda \in \mathbb{R}^{+}$, where $m$ is the complex dimension, since rescaling a Kähler metric by $g \rightsquigarrow \lambda g$ results in $\omega \rightsquigarrow \lambda \omega, s \rightsquigarrow \lambda^{-1} s, f \rightsquigarrow \lambda f$, and $d \mu \rightsquigarrow \lambda^{m} d \mu$. By taking $\lambda$ to be an arbitrary positive rational, we therefore see that $\mathfrak{F}(\Xi, \Omega)=0$ whenever $\Xi \in \mathfrak{r}_{\mathfrak{u}}$ and $\Omega \in H^{2}(M, \mathbb{Q}) \cap \mathcal{K}$, where $\mathcal{K}$ once again 
denotes the Kähler cone. However, for any fixed $\Xi$, the right-hand-side of (2.1) clearly depends smoothly on the Kähler metric $g$, and $\mathfrak{F}(\Xi, \Omega)$ therefore is a smooth function of the Kähler class $\Omega$. But $h^{2,0}(M)=0$ implies that $H^{2}(M, \mathbb{Q}) \cap \mathcal{K}$ is dense in $\mathcal{K}$. Thus, for any $\Xi \in \mathfrak{r}_{\mathfrak{u}}$, we have shown that $\mathfrak{F}(\Xi, \Omega)=0$ for a dense set of $\Omega \in \mathcal{K}$. Continuity therefore implies that $\mathfrak{F}(\Xi, \Omega)=0$ for all $\Omega \in \mathcal{K}$. Hence $\mathfrak{F}(\Omega) \in \mathfrak{h}^{*}$ annihilates $\mathfrak{r}_{\mathfrak{u}}$ for any Kähler class $\Omega$ on $M$.

Under the hypotheses of Proposition A.3, we can thus view $\mathfrak{F}(\Omega)$ as belonging to the complexified Lie coalgebra $\mathfrak{g}_{\mathbb{C}}^{*}=\mathfrak{g}^{*} \otimes \mathbb{C}$ of a maximal compact subgroup $\mathbf{G} \subset \mathbf{H}$. By averaging, let us now represent our given Kähler class $\Omega$ by a G-invariant Kähler metric $g$. The Lie algebra of Killing fields of $g$ then can be identified with the real holomorphy potentials of integral 0, which are their Hamiltonians; the Lie bracket on $\mathfrak{g}$ then becomes the Poisson bracket $\{\cdot, \cdot\}$ on Hamiltonians. Since the scalar curvature $s$ of $g$ is also a real function, formula $(2.1)$ thus tells us that $\mathfrak{F}(\Omega)$ is actually a real linear functional on $\mathfrak{g}$; that is, $\mathfrak{F}(\Omega) \in \mathfrak{g}^{*}$. This proves Proposition 2.1.

\section{References}

[1] M. Abreu, Kähler geometry of toric varieties and extremal metrics, Internat. J. Math. 9 (1998) 641-651.

[2] C. Arezzo, F. Pacard, and M. Singer, Extremal metrics on blowups, Duke Math. J. 157 (2011) 1-51.

[3] M. F. Атіуан, Convexity and commuting Hamiltonians, Bull. London Math. Soc. 14 (1982) 1-15.

[4] T. Aubin, Équations du type Monge-Ampère sur les variétés kähleriennes compactes, C. R. Acad. Sci. Paris Sér. A-B 283 (1976) Aiii, A119-A121.

[5] R. BACH, Zur Weylschen Relativitätstheorie und der Weylschen Erweiterung des Krümmungstensorbegriffs., Math. Zeitschr. 9 (1921) 110-135.

[6] S. BAndo, An obstruction for Chern class forms to be harmonic, Kodai Math. J. 29 (2006) 337-345.

[7] W. Barth, C. Peters, and A. VAn de Ven, Compact complex surfaces, volume 4 of Ergebnisse der Mathematik und ihrer Grenzgebiete (3), Springer-Verlag, Berlin, 1984.

[8] L. BÉRARD-BERGERY, Sur de nouvelles variétés riemanniennes d'Einstein, in Institut Élie Cartan, 6, volume 6 of Inst. Élie Cartan, pp. 1-60, Univ. Nancy, Nancy, 1982.

[9] A. L. BEsse, Einstein manifolds, volume 10 of Ergebnisse der Mathematik und ihrer Grenzgebiete (3), Springer-Verlag, Berlin, 1987.

[10] N. Buchdahl, On compact Kähler surfaces, Ann. Inst. Fourier (Grenoble) 49 (1999) 287-302.

[11] E. Calabi, Extremal Kähler metrics, in Seminar on Differential Geometry, volume 102 of Ann. Math. Studies, pp. 259-290, Princeton Univ. Press, Princeton, N.J., 1982.

[12] E. CALABI, Extremal Kähler metrics. II, in Differential Geometry and Complex Analysis, pp. 95-114, Springer, Berlin, 1985.

[13] X. X. Chen, Space of Kähler metrics. III. On the lower bound of the Calabi energy and geodesic distance, Invent. Math. 175 (2009) 453-503.

[14] X. X. Chen and G. Tian, Geometry of Kähler metrics and foliations by holomorphic discs, Publ. Math. Inst. Hautes Études Sci. (2008) 1-107.

[15] X. Chen, C. LeBrun, and B. Weber, On conformally Kähler, Einstein manifolds, J. Amer. Math. Soc. 21 (2008) 1137-1168. 
[16] B. Chen, A.-M. Li, and L. Sheng, Extremal Metrics on Toric Surfaces, e-print, arXiv:1008.2607v3 [math.DG], 2010.

[17] C. Chevalley, Théorie des groupes de Lie. Tome III. Théorèmes généraux sur les algèbres de Lie, Actualités Sci. Ind. no. 1226, Hermann \& Cie, Paris, 1955.

[18] T. Delzant, Hamiltoniens périodiques et images convexes de l'application moment, Bull. Soc. Math. France 116 (1988) 315-339.

[19] A. Derdziński, Self-dual Kähler manifolds and Einstein manifolds of dimension four, Compositio Math. 49 (1983) 405-433.

[20] S. K. Donaldson, Scalar curvature and projective embeddings. I, J. Differential Geom. 59 (2001) 479-522.

[21] S. K. Donaldson, Scalar curvature and stability of toric varieties, J. Differential Geom. 62 (2002) 289-349.

[22] A. FuJiki, On automorphism groups of compact Kähler manifolds, Invent. Math. 44 (1978) 225-258.

[23] W. Fulton, Introduction to toric varieties, volume 131 of Annals of Mathematics Studies, Princeton University Press, Princeton, NJ, 1993.

[24] A. FutAKI, An obstruction to the existence of Einstein Kähler metrics, Invent. Math. 73 (1983) 437-443.

[25] A. Futaki and T. MABuchi, Uniqueness and periodicity of extremal Kähler vector fields, in Proceedings of GARC Workshop on Geometry and Topology '93 (Seoul, 1993), volume 18 of Lecture Notes Ser., pp. 217-239, Seoul, 1993, Seoul Nat. Univ.

[26] P. Griffiths and J. HARris, Principles of Algebraic Geometry, Wiley-Interscience, New York, 1978.

[27] V. Guillemin, Moment maps and combinatorial invariants of Hamiltonian $T^{n}$ spaces, volume 122 of Progress in Mathematics, Birkhäuser Boston Inc., Boston, MA, 1994.

[28] V. Guillemin and S. Sternberg, Convexity properties of the moment mapping, Invent. Math. 67 (1982) 491-513.

[29] S. W. Hawking, C. J. Hunter, and D. N. Page, NUT charge, anti-de Sitter space, and entropy, Phys. Rev. D (3) 59 (1999) 044033, 6.

[30] A. D. Hwang and S. R. Simanca, Extremal Kähler metrics on Hirzebruch surfaces which are locally conformally equivalent to Einstein metrics, Math. Ann. 309 (1997) 97-106.

[31] J. Kim, C. LeBrun, and M. Pontecorvo, Scalar-flat Kähler surfaces of all genera, J. Reine Angew. Math. 486 (1997) 69-95.

[32] S. Kobayashi, Fixed points of isometries, Nagoya Math. J. 13 (1958) 63-68.

[33] K. Kodaira, On compact complex analytic surfaces. I, Ann. of Math. (2) 71 (1960) $111-152$.

[34] C. LeBrun, Explicit self-dual metrics on $\mathbb{C P}_{2} \# \cdots \# \mathbb{C P}_{2}$, J. Differential Geom. 34 (1991) 223-253.

[35] C. LeBrun, Anti-self-dual metrics and Kähler geometry, in Proceedings of the International Congress of Mathematicians, Vol. 1, 2 (Zürich, 1994), pp. 498-507, Basel, 1995, Birkhäuser.

[36] C. LeBrun, Einstein metrics on complex surfaces, in Geometry and Physics (Aarhus, 1995), volume 184 of Lecture Notes in Pure and Appl. Math., pp. 167-176, Dekker, New York, 1997.

[37] C. LeBrun, Einstein Manifolds and Extremal Kähler Metrics, to appear in Crelle; e-print arXiv:1009.1270 [math.DG], 2010.

[38] C. LeBrun, On Einstein, Hermitian 4-Manifolds, J. Differential Geom. 90 (2012) 277-302.

[39] C. LeBrun and S. R. Simanca, On the Kähler classes of extremal metrics, in Geometry and Global Analysis (Sendai, 1993), pp. 255-271, Tohoku Univ., Sendai, 1993. 
[40] C. LeBrun and S. R. SimancA, Extremal Kähler metrics and complex deformation theory, Geom. Funct. Anal. 4 (1994) 298-336.

[41] T. Mabuchi, Einstein-Kähler forms, Futaki invariants and convex geometry on toric Fano varieties, Osaka J. Math. 24 (1987) 705-737.

[42] T. Mabuchi, An algebraic character associated with the Poisson brackets, in Recent topics in differential and analytic geometry, volume 18 of Adv. Stud. Pure Math., pp. 339-358, Academic Press, Boston, MA, 1990.

[43] T. MABUChI, Uniqueness of extremal Kähler metrics for an integral Kähler class, Internat. J. Math. 15 (2004) 531-546.

[44] J. Milnor, Morse Theory, volume 51 of Ann. Math. Studies, Princeton University Press, Princeton, N.J., 1963, Based on lecture notes by M. Spivak and R. Wells.

[45] Y. Nakagawa, Bando-Calabi-Futaki characters of Kähler orbifolds, Math. Ann. 314 (1999) 369-380.

[46] Y. NAKagawa, Bando-Calabi-Futaki character of compact toric manifolds, Tohoku Math. J. (2) $\mathbf{5 3}$ (2001) 479-490.

[47] Y. Odaka, C. Spotti, and S. Sun, Compact Moduli Spaces of Del Pezzo Surfaces and Kähler-Einstein Metrics, e-print arXiv:1210.0858 [math.DG], 2012.

[48] D. PAGe, A Compact Rotating Gravitational Instanton, Phys. Lett. 79B (1979) 235238.

[49] E. Shelukhin, Remarks on invariants of Hamiltonian loops, J. Topol. Anal. 2 (2010) $277-325$.

[50] Y. Siu, Every K3 Surface is Kähler, Inv. Math. 73 (1983) 139-150.

[51] C. H. TAubes, The existence of anti-self-dual conformal structures, J. Differential Geom. 36 (1992) 163-253.

[52] G. Tian, On Calabi's conjecture for complex surfaces with positive first Chern class, Invent. Math. 101 (1990) 101-172.

[53] G. Tian, Kähler-Einstein metrics on algebraic manifolds, in Transcendental methods in algebraic geometry (Cetraro, 1994), volume 1646 of Lecture Notes in Math., pp. 143-185, Springer, Berlin, 1996.

[54] S. T. YAU, On the Ricci curvature of a compact Kähler manifold and the complex Monge-Ampère equation. I, Comm. Pure Appl. Math. 31 (1978) 339-411.

Department of Mathematics, SUny at Stony Brook, Stony Brook, Ny 11794-3651, USA

E-mail address: claude@math.sunysb.edu 